\title{
Relaxation and simulation of a barotropic three-phase flow model
}

\author{
Hamza Boukili ${ }^{1,2}$, Jean-Marc Hérard ${ }^{1,2}$ \\ 1 EDF Lab Chatou, 6 quai Watier, 78400 Chatou \\ 2 I2M, Aix Marseille Université, 39 rue Joliot Curie, 13453 Marseille
}

\begin{abstract}
We focus here on a three-phase flow model in order to represent complex flows involving liquid metal droplets, liquid water, and its vapour. The governing equations and its main properties are given, and focus is given on the pressurevelocity relaxation process on the one hand, and on the structure of solutions of the one-dimensional Riemann problem associated with pure convective effects. A fractional step method that computes successively the convective part and the relaxation effects is used to obtain approximate solutions on unstructured meshes. Details of algorithms are provided, and it is shown that the numerical method preserves positive values of statistical fractions and partial masses. Verification and validation test cases are presented, and some perspectives are eventually drawn.
\end{abstract}

Hamza Boukili $^{1,2}$, Jean-Marc Hérard ${ }^{1,2}$

${ }^{1}$ EDF Lab Chatou, 6, quai Watier, 78400, Chatou, France

${ }^{2}$ I2M, Aix Marseille Université, 39 rue Joliot Curie, 13453, Marseille, France

e-mail: hamza.boukili@edf.fr,jean-marc.herard@edf.fr 


\section{Introduction}

The modeling and the accurate simulation of steam explosion is actually an open topic; it is indeed important for nuclear safety analysis, and it deserves relevant and meaningful models, and also appropriate numerical methods, to get decent approximations of pressure waves acting on solid structures. The steam explosion may occur when some very hot liquid metal flows down in a quiet liquid component such as water. In that case the heat transfer towards the water component depends on the local structure of liquid metal droplets. The liquid water surrounding the liquid metal droplet is suddenly changed into steam, and this, in turn, can modify the process, since the thin layer of steam in the vicinity of the metal droplet can inhibit the heat transfer between the two components. Afterwards, pressure waves may propagate, and change the topology of the flow, because droplets are sheared and dislocated when high relative velocities are involved. The break-up of liquid metal droplets thus increases the interfacial area between droplets and liquid water, so that the heat transfer may again develop and feed-up the whole chain. Different phenomenological scenarios have been proposed in the literature to predict these flow patterns. We refer the reader to [4] and references therein in order to have a better understanding of that problem, and also to the recent paper [28].

An important point to quote at once is that the sudden increase of vapour concentration results in huge pressure waves including shock and rarefaction waves. Another feature is that the three fields (liquid metal droplets, liquid water and steam) are immiscible; moreover, mass transfer may only happen within the water component, more precisely between the liquid water and its vapour phase. Some multiphase flow models have been proposed in the past, which mainly rely on the instantaneous pressure relaxation assumption. However, as it is well known for standard two-phase flow models, one straightforward drawback is that associated sets of PDEs are not hyperbolic. Even more, jump relations through -expected- shock waves are not defined. This may not only result in several difficulties when tackling the steam explosion description, in order to estimate the amplitude of shock waves on wall boundaries, since we need to compute an initial-value problem, but it also renders the estimation of the pressure shock waves questionable.

Hence the work presented herein aims at providing some original preliminary results in the direction of steam explosion modeling. For that purpose, we first need to define adequate sets of PDEs, so that the following requirements arise:

- the whole model must comply with hyperbolicity requirements;

- some physical entropy inequality is needed;

- unique jump conditions are mandatory;

and of course, schemes should provide convergent approximations of pressure shock waves. 
Obviously, there are several difficulties on the way to such a correct and physically relevant description. In order to simplify a little bit the whole approach, we will focus in this work on barotropic situations, thus neglecting heat transfer. One basic reason is that one needs to get some estimation of the interfacial area, since this seems to be one of the key points on the way to pave towards a correct modeling of steam explosion. However, we emphasize that extensions of the barotropic three-phase model that will be considered here [21] already exist (see [20]), which means that the sequel of the present framework is indeed clear, at least from a global point of view. In other words, the current barotropic model [21] may be seen as a rough version of model [20]. It is also worth noting that the latter models $[21,20]$ are actually quite similar to the classical two-phase flow models arising from $[3,5,25,16,9]$.

Hence, the present paper will be organized as follows. We will first present the governing set of equations for the basic barotropic three-phase flow model, and then give a slight extension in order to account for the interfacial area. Afterwards, we will give the main properties of both of these, while focusing on solutions of the one-dimensional Riemann problem. This enables to extract meaningful analytical test cases including shock waves, rarefaction waves and contact discontinuities. Next, we will briefly discuss the inner pressure-velocity relaxation processes, and comment the whole. The second part will be devoted to the presentation of a fractional step method that treats separately convective effects and source terms. In this paper, we will only consider a rough Finite Volume scheme to cope with the convective part, and postpone the derivation of more accurate -though stable- approximate Riemann solvers to a future work; this will enable us to concentrate on the numerical treatment of velocity and pressure relaxation effects. The first section in the last part will provide some approximate solutions of analytical test cases, including the measure of the $L^{1}$ norm of the error. Eventually, we will show some results corresponding to the propagation of a shock wave through a cloud of liquid droplets, which corresponds to the experimental setup of the paper [8]. One crucial point is that the latter configuration is very similar to what happens in the steam explosion setup, and meanwhile, this will give some good confidence with respect to the modeling of the break-up phenomenon. The conclusion will highlight the main directions of progress towards a complete simulation of steam explosion. 


\section{Governing equations and main properties of the two-dimensional three-phase flow model}

\subsection{Governing equations}

Throughout the paper, $\alpha_{k} \in[0,1], \rho_{k}, m_{k}=\alpha_{k} \rho_{k}, \mathbf{U}_{\mathbf{k}}$, will denote the mean statistical fraction, the mean density, the partial mass and the mean velocity of phase $k$ Statistical fractions are such that:

$$
\alpha_{1}+\alpha_{2}+\alpha_{3}=1
$$

The mean pressure $P_{k}\left(\rho_{k}\right)$ is an increasing function which is such that:

$$
\lim _{x \rightarrow \infty} P_{k}(x)=+\infty \quad ; \quad \lim _{x \rightarrow 0} P_{k}(x)=0
$$

and we introduce the phasic speed of sound waves $c_{k}$ such that $: c_{k}^{2}=P_{k}^{\prime}\left(\rho_{k}\right)$.

The state variable $\mathbf{W}$ will denote the following vector:

$$
\mathbf{W}=\left(\alpha_{2}, \alpha_{3}, m_{1}, m_{2}, m_{3}, m_{1} \mathbf{U}_{\mathbf{1}}, m_{2} \mathbf{U}_{\mathbf{2}}, m_{3} \mathbf{U}_{\mathbf{3}}\right)^{t}
$$

The set of governing equations for the three-phase flow model will read ([21]):

$$
\left\{\begin{array}{l}
\frac{\partial \alpha_{k}}{\partial t}+\mathscr{V}_{\mathbf{i}}(\mathbf{W}) \nabla \alpha_{k}=\phi_{k}(\mathbf{W}) \\
\frac{\partial m_{k}}{\partial t}+\nabla \cdot\left(m_{k} \mathbf{U}_{\mathbf{k}}\right)=0 \\
\frac{\partial m_{k} \mathbf{U}_{\mathbf{k}}}{\partial t}+\nabla \cdot\left(m_{k} \mathbf{U}_{\mathbf{k}} \times \mathbf{U}_{\mathbf{k}}+\alpha_{k} P_{k} \mathbf{I d}\right)+\Sigma_{l=1, l \neq k}^{3} \Pi_{k l}(\mathbf{W}) \nabla \alpha_{l}=m_{k} \mathrm{~S}_{\mathbf{k}}(\mathbf{W})
\end{array}\right.
$$

Source terms should be such that:

$$
\Sigma_{k=1}^{3} m_{k} \mathrm{~S}_{\mathrm{k}}(\mathbf{W})=\mathbf{0}
$$

and:

$$
\sum_{k=1}^{3} \phi_{k}(\mathbf{W})=0
$$

since they only take into account transfers between the different phases.

Moreover, a similar constraint holds for the $\Pi_{k l}(\mathbf{W})$ since:

$$
\Sigma_{k=1}^{3}\left(\Sigma_{l=1, l \neq k}^{3} \Pi_{k l}(\mathbf{W}) \nabla \alpha_{l}\right)=\mathbf{0}
$$

and of course, owing to (1), the three quantities $\nabla \alpha_{l}$ are in agreement with: 


$$
\Sigma_{k=1}^{3} \nabla \alpha_{k}=\mathbf{0}
$$

The first and second equations provide the evolution of the statistical fraction and mass fraction within phase $k$, while the third equation stands for the momentum balance equation. The interface velocity $\mathscr{V}_{\mathbf{i}}(\mathbf{W})$ and the interfacial pressures $\Pi_{k l}(\mathbf{W})$ are not unknown. Actually the interface pressures are uniquely defined as functions of the interface velocity. More precisely, if $\mathscr{Y}_{\mathbf{i}}(\mathbf{W})$ denotes a convex combination of the phasic velocities, that is :

$$
\mathscr{V}_{\mathbf{i}}(\mathbf{W})=a_{1}(\mathbf{W}) \mathbf{U}_{\mathbf{1}}+a_{2}(\mathbf{W}) \mathbf{U}_{\mathbf{2}}+a_{3}(\mathbf{W}) \mathbf{U}_{\mathbf{3}}
$$

where all $a_{k}(\mathbf{W})$ lie in $[0,1]$, with the constraint: $a_{1}(\mathbf{W})+a_{2}(\mathbf{W})+a_{3}(\mathbf{W})=1$, then all interfacial pressures should be such that:

$$
\left\{\begin{array}{l}
\Pi_{12}(\mathbf{W})=\left(1-a_{1}(\mathbf{W})\right) P_{1}+a_{1}(\mathbf{W}) P_{2} \\
\Pi_{21}(\mathbf{W})=a_{2}(\mathbf{W}) P_{1}+\left(1-a_{2}(\mathbf{W})\right) P_{2} \\
\Pi_{13}(\mathbf{W})=\left(1-a_{1}(\mathbf{W})\right) P_{1}+a_{1}(\mathbf{W}) P_{3} \\
\Pi_{31}(\mathbf{W})=a_{3}(\mathbf{W}) P_{1}+\left(1-a_{3}(\mathbf{W})\right) P_{3} \\
\Pi_{23}(\mathbf{W})=\left(1-a_{2}(\mathbf{W})\right) P_{2}+a_{2}(\mathbf{W}) P_{3} \\
\Pi_{32}(\mathbf{W})=a_{3}(\mathbf{W}) P_{2}+\left(1-a_{3}(\mathbf{W})\right) P_{3}
\end{array}\right.
$$

These closure laws, together with a correct choice of the right hand side contributions $\phi_{k}(\mathbf{W})$ and $\mathrm{S}_{\mathrm{k}}(\mathbf{W})$, will ensure that smooth solutions of system (3) would comply with a relevant entropy balance.

Closure laws to account for drag effects between phases $k, l$ are simply chosen as:

$$
m_{k} \mathrm{~S}_{\mathbf{k}}(\mathbf{W})=\Sigma_{l=1}^{3} e_{k l}(\mathbf{W})\left(\mathbf{U}_{\mathbf{l}}-\mathbf{U}_{\mathbf{k}}\right)
$$

where the symetric positive functions $e_{k l}(\mathbf{W})=e_{l k}(\mathbf{W})$ are chosen in agreement with the two-phase flow literature.

Meanwhile, we define:

$$
\phi_{k}(\mathbf{W})=\Sigma_{l=1}^{3} d_{k l}(\mathbf{W})\left(P_{k}-P_{l}\right)
$$

where the symetric positive functions $d_{k l}(\mathbf{W})=d_{l k}(\mathbf{W})$, which involve presssure relaxation time scales, will be taken from [15].

\subsection{Main properties of the three-phase flow model}

We introduce $\psi_{k}\left(\rho_{k}\right)$ such that $\psi_{k}^{\prime}\left(\rho_{k}\right)=\frac{P_{k}\left(\rho_{k}\right)}{\rho_{k}^{2}}$, and define the mixture entropy $\eta(\mathbf{W})$ and the entropy flux $\mathbf{f}_{\eta}(\mathbf{W})$ as: 


$$
\left\{\begin{array}{l}
\eta(\mathbf{W})=\Sigma_{k=1}^{3} m_{k}\left(\left|\mathbf{U}_{\mathbf{k}} \cdot \mathbf{U}_{\mathbf{k}}\right| / 2+\psi_{k}\left(\rho_{k}\right)\right) \\
\mathbf{f}_{\eta}(\mathbf{W})=\Sigma_{k=1}^{3} m_{k}\left(\left|\mathbf{U}_{\mathbf{k}} \cdot \mathbf{U}_{\mathbf{k}}\right| / 2+\psi_{k}\left(\rho_{k}\right)+\frac{P_{k}}{\rho_{k}}\right) \mathbf{U}_{\mathbf{k}}
\end{array}\right.
$$

Owing to the closure laws (5) and (6), the following property holds for smooth solutions of (3):

\section{Property 1:}

Smooth solutions of (3) comply with the following entropy inequality:

$$
\frac{\partial \eta(\mathbf{W})}{\partial t}+\nabla \cdot\left(\mathbf{f}_{\eta}(\mathbf{W})\right) \leq 0 .
$$

(see [21]).

Now, using invariance under frame rotation of system (3), introducing two normal vectors $\mathbf{n}=\left(n_{x}, n_{y}\right)$ and $\tau=\left(-n_{y}, n_{x}\right)$ in $\mathscr{R}^{2}$, such that: $n_{x}^{2}+n_{y}^{2}=1$, and rewriting (3) in the (n, $\tau)$ frame, we may consider the one-dimensional homogeneous system in the $\mathbf{n}$ direction obtained by neglecting transverse variations $\frac{\partial g}{\partial \tau}$ with respect to $\tau$, whatever $g$ is. Thus we get:

$$
\left\{\begin{array}{l}
\frac{\partial \alpha_{k}}{\partial t}+\mathscr{Y}_{\mathbf{i}}(\mathbf{W}) \cdot \mathbf{n} \frac{\partial \alpha_{k}}{\partial n}=0 ; \\
\frac{\partial m_{k}}{\partial t}+\frac{\partial m_{k} \mathbf{U}_{\mathbf{k}} \cdot \mathbf{n}}{\partial n}=0 ; \\
\frac{\partial m_{k} \mathbf{U}_{\mathbf{k}} \cdot \mathbf{n}}{\partial t}+\frac{\partial m_{k}\left(\mathbf{U}_{\mathbf{k}} \cdot \mathbf{n}\right)^{2}+\alpha_{k} P_{k}}{\partial n}+\Sigma_{l=1, l \neq k}^{3} \Pi_{k l}(\mathbf{W}) \frac{\partial \alpha_{l}}{\partial n}=0 ; \\
\frac{\partial m_{k} \mathbf{U}_{\mathbf{k}} \cdot \tau}{\partial t}+\frac{\partial m_{k}\left(\mathbf{U}_{\mathbf{k}} \cdot \mathbf{n}\right)\left(\mathbf{U}_{\mathbf{k}} \cdot \tau\right)}{\partial n}=0 .
\end{array}\right.
$$

A straightforward consequence is the following:

\section{Property 2:}

System (9) is hyperbolic. It admits the following real eigenvalues:

$$
\lambda_{0,1}(\mathbf{W})=\mathscr{Y}_{\mathbf{i}}(\mathbf{W}) \cdot \mathbf{n} \quad ; \quad \lambda_{2-7}(\mathbf{W})=\mathbf{U}_{\mathbf{k}} \cdot \mathbf{n} \pm c_{k} \quad ; \quad \lambda_{8-10}(\mathbf{W})=\mathbf{U}_{\mathbf{k}} \cdot \mathbf{n}
$$

and associated right-eigenvectors span the whole space $I R^{11}$ unless:

$$
\left(\mathscr{V}_{\mathbf{i}}(\mathbf{W})-\mathbf{U}_{\mathbf{k}}\right) \cdot \mathbf{n}= \pm c_{k} .
$$

The proof is classical and left to the reader.

In the sequel of the paper, we will focus on the following closure law:

$$
\mathscr{V}_{\mathbf{i}}(\mathbf{W})=\mathbf{U}_{\mathbf{1}}
$$


which implies :

$$
\left\{\begin{array}{l}
\Pi_{12}(\mathbf{W})=\Pi_{21}(\mathbf{W})=\Pi_{23}(\mathbf{W})=P_{2} \\
\Pi_{13}(\mathbf{W})=\Pi_{31}(\mathbf{W})=\Pi_{32}(\mathbf{W})=P_{3}
\end{array}\right.
$$

owing to (4). We note at a glance that this is in fact the straightforward counterpart of the Baer-Nunziato closure laws in a two-phase framework ([3]).

\subsection{Some additional results in the one-dimensional framework}

In a pure one-dimensional framework, we may focus on the structure of the latter fields. More precisely, if we consider:

$$
\left\{\begin{array}{l}
\frac{\partial \alpha_{k}}{\partial t}+\mathscr{V}_{i}(W) \frac{\partial \alpha_{k}}{\partial x}=0 \\
\frac{\partial m_{k}}{\partial t}+\frac{\partial m_{k} u_{k}}{\partial x}=0 \\
\frac{\partial m_{k} u_{k}}{\partial t}+\frac{\partial m_{k} u_{k}^{2}+\alpha_{k} P_{k}}{\partial x}+\Sigma_{l=1, l \neq k}^{3} \Pi_{k l}(\mathbf{W}) \frac{\partial \alpha_{l}}{\partial x}=0
\end{array} .\right.
$$

we may set : $\mathbf{X}=\left(\alpha_{2}, \alpha_{3}, m_{1}, m_{2}, m_{3}, m_{1} u_{1}, m_{2} u_{2}, m_{3} u_{3}\right)$, and we get at once:

\section{Property 3:}

- The convective subset (13) admits eight real eigenvalues which read:

$$
\lambda_{0,1}(\mathbf{X})=u_{1} \quad ; \quad \lambda_{2-7}(\mathbf{X})=u_{k} \pm c_{k} .
$$

The field associated with eigenvalues $\lambda_{0,1}(\mathbf{X})$ is linearly degenerate. Meanwhile, fields associated with $\lambda_{2-7}(\mathbf{X})$ are genuinely non linear.

- The six Riemann invariants in the $0-1$ coupling wave write:

$$
\begin{gathered}
I_{0,1}^{1}(\mathbf{X})=u_{1} \quad ; \quad I_{0,1}^{2}(\mathbf{X})=m_{2}\left(u_{2}-u_{1}\right) \quad ; \quad I_{0,1}^{3}(\mathbf{X})=m_{3}\left(u_{3}-u_{1}\right) ; \\
I_{0,1}^{4}(\mathbf{X})=\frac{\left(u_{1}-u_{2}\right)^{2}}{2}+\int_{0}^{\rho_{2}} \frac{c_{2}^{2}(x)}{x} d x \quad ; \quad I_{0,1}^{5}(\mathbf{X})=\frac{\left(u_{1}-u_{3}\right)^{2}}{2}+\int_{0}^{\rho_{3}} \frac{c_{3}^{2}(x)}{x} d x ; \\
I_{0,1}^{6}(\mathbf{X})=\Sigma_{k=1}^{3}\left(\alpha_{k} P_{k}\right)+m_{2}\left(u_{2}-u_{1}\right)^{2}+m_{3}\left(u_{3}-u_{1}\right)^{2} .
\end{gathered}
$$

- Within each isolated GNL wave, unique jump conditions between $\mathbf{X}_{l}, \mathbf{X}_{r}$ states arise: 


$$
\left\{\begin{array}{l}
{\left[\alpha_{k}\right]_{l}^{r}=0 ;} \\
-\sigma\left[m_{k}\right]_{l}^{r}+\left[m_{k} u_{k}\right]_{l}^{r}=0 ; \\
-\sigma\left[m_{k} u_{k}\right]_{l}^{r}+\left[m_{k} u_{k}^{2}+\alpha_{k} P_{k}\right]_{l}^{r}=0 ;
\end{array}\right.
$$

where $\sigma$ denotes the speed of the discontinuity separating states $\mathbf{X}_{l}, \mathbf{X}_{r}$.

- Within the GNL wave associated with $\lambda_{2}(\mathbf{X})=u_{1}-c_{1}\left(\right.$ resp. $\left.\lambda_{3}(\mathbf{X})=u_{1}+c_{1}\right)$, Riemann invariants connecting $\mathbf{X}_{l}, \mathbf{X}_{r}$ states are $\left(\alpha_{2}, \alpha_{3}, \rho_{2}, \rho_{3}, u_{2}, u_{3}\right)$ and: $u_{1}+$ $\int \frac{c_{1}\left(\rho_{1}\right)}{\rho_{1}} d \rho_{1}$ (resp. $\left.u_{1}-\int \frac{c_{1}\left(\rho_{1}\right)}{\rho_{1}} d \rho_{1}\right)$. Similar results hold for $4-5$ and $6-7$ fields.

The latter Riemann invariants and jump conditions will enable us to construct exact solutions in order to check the true convergence of algorithms (see for instance the first two test cases in section 4). Again, the latter property is the straightforward counterpart of what occurs when focusing on the two-phase barotropic BaerNunziato model (see [3]).

Actually, if $\mathbf{X}_{l}$ denotes the state on the left-hand side of the $0-1$ coupling wave, and if we assume that $\left(\alpha_{2}\right)_{r},\left(\alpha_{3}\right)_{r}$ are also given, we may compute the right state $\mathbf{X}_{r}$ by enforcing:

$$
I_{0,1}^{m}\left(\mathbf{X}_{r}\right)=I_{0,1}^{m}\left(\mathbf{X}_{l}\right)
$$

where the left state is given. We only need to compute $x_{2}=\left(\rho_{2}\right)_{r}$ solution of the scalar equation $g_{2}\left(x_{2}\right)=0$ where:

$$
g_{2}\left(x_{2}\right)=\frac{\left(I_{0,1}^{2}\left(\mathbf{X}_{l}\right)\right)^{2}}{2\left(\left(\alpha_{2}\right)_{r} x_{2}\right)^{2}}+\int_{0}^{x_{2}} \frac{c_{2}^{2}(x)}{x} d x-I_{0,1}^{4}\left(\mathbf{X}_{l}\right)
$$

and in a similar way $x_{3}=\left(\rho_{3}\right)_{r}$ solution of the scalar equation $g_{3}\left(x_{3}\right)=0$ where:

$$
g_{3}\left(x_{3}\right)=\frac{\left(I_{0,1}^{3}\left(\mathbf{X}_{l}\right)\right)^{2}}{2\left(\left(\alpha_{3}\right)_{r} x_{3}\right)^{2}}+\int_{0}^{x_{3}} \frac{c_{3}^{2}(x)}{x} d x-I_{0,1}^{5}\left(\mathbf{X}_{l}\right)
$$

Once these two scalar quantities have been calculated, we may update $\left(u_{2}\right)_{r}$ and $\left(u_{3}\right)_{r}$ using the following equalities:

$$
\left(u_{2}\right)_{r}=\left(u_{1}\right)_{l}+\frac{I_{0,1}^{2}\left(\mathbf{X}_{l}\right)}{\left(m_{2}\right)_{r}}
$$

and also:

$$
\left(u_{3}\right)_{r}=\left(u_{1}\right)_{l}+\frac{I_{0,1}^{3}\left(\mathbf{X}_{l}\right)}{\left(m_{3}\right)_{r}}
$$

The last unknown $x_{1}=\left(\rho_{1}\right)_{r}$ is found by solving :

$$
I_{0,1}^{6}\left(x_{1}\right)=I_{0,1}^{6}\left(\mathbf{X}_{l}\right)
$$


with some abuse of notation.

We also note that the form of the function $g_{k}(x)$ is similar to the one encountered in the shallow-water framework, when the "bottom of the lake" is not uniform. The latter results will be used in section 4.1. Other technical results on the convexity of the entropy and on the symmetric form of the system can be found in [23].

\subsection{A slightly modified three-phase flow model}

For some practical purposes, we will need some enrichment of the latter three-phase flow model (3), in order to account for interfacial area modeling. Actually this will be used in order to compute situations where droplets of liquid phase 1 are sheared and dislocated.

Hence, if $A$ denotes some -positive- function standing for the interfacial area, we introduce some extension of the latter three-phase flow model (3), which reads:

$$
\left\{\begin{array}{l}
\frac{\partial A}{\partial t}+\nabla \cdot\left(A \mathbf{U}_{\mathbf{1}}\right)=g(A, \mathbf{W}) \\
\frac{\partial \alpha_{k}}{\partial t}+\mathscr{Y}_{\mathbf{i}}(\mathbf{W}) \nabla \alpha_{k}=\phi_{k}(\mathbf{W}) \\
\frac{\partial m_{k}}{\partial t}+\nabla \cdot\left(m_{k} \mathbf{U}_{\mathbf{k}}\right)=0 ; \\
\frac{\partial m_{k} \mathbf{U}_{\mathbf{k}}}{\partial t}+\nabla \cdot\left(m_{k} \mathbf{U}_{\mathbf{k}} \times \mathbf{U}_{\mathbf{k}}+\alpha_{k} P_{k} \mathbf{I} \mathbf{d}\right)+\Sigma_{l=1, l \neq k}^{3} \Pi_{k l}(\mathbf{W}) \nabla \alpha_{l}=m_{k} \mathrm{~S}_{\mathbf{k}}^{\mathrm{A}}(\mathbf{A}, \mathbf{W}) .
\end{array}\right.
$$

or in a more condensed form:

$$
\left\{\begin{array}{l}
\frac{\partial A}{\partial t}+\nabla \cdot\left(A \mathbf{U}_{\mathbf{1}}\right)=g(A, \mathbf{W}) \\
\frac{\partial \mathbf{W}}{\partial t}+\nabla \cdot(\mathbf{F}(\mathbf{W}))+\Sigma_{l=1}^{3} \mathbf{G}_{\mathbf{l}}(\mathbf{W}) \nabla \alpha_{l}=\mathbf{r}(\mathbf{A}, \mathbf{W}) .
\end{array}\right.
$$

where the non-negative function $g(A, \mathbf{W})$, which takes dislocation effects of phase 1 droplets into account, will be described in detail afterwards. The first governing equation for $A$ is taken from $[28,30]$. Actually, the closure laws for $\Pi_{k l}(\mathbf{W})$ interfacial terms still agree with (4). Furthermore, we assume that we still have:

$$
m_{k} \mathrm{~S}_{\mathrm{k}}^{\mathbf{A}}(\mathbf{A}, \mathbf{W})=\Sigma_{l=1}^{3} e_{k l}^{A}(A, \mathbf{W})\left(\mathbf{U}_{\mathbf{l}}-\mathbf{U}_{\mathbf{k}}\right)
$$

where the positive functions $e_{k l}^{A}(A, \mathbf{W})=e_{l k}^{A}(A, \mathbf{W})$ are directly deduced from the latter $e_{k l}(\mathbf{W})$. 
Then, if we choose the combination $a_{1}=1, a_{2}=a_{3}=0$, we get the following result:

Proposition 1:

- The homogeneous convective part of system (17) admits real eigenvalues; it is hyperbolic unless:

$$
\left|\left(\mathbf{U}_{1}-\mathbf{U}_{\mathbf{k}}\right) \cdot \mathbf{n}\right|=c_{k}
$$

- Smooth solutions of (17) comply with :

$$
\frac{\partial \eta(\mathbf{W})}{\partial t}+\nabla \cdot\left(\mathbf{f}_{\eta}(\mathbf{W})\right)=R H S_{\eta}(\mathbf{W}) \leq 0 .
$$

Proof: the computation of eigenvalues is classical. Turning to the entropy inequality, the only difference with the former three-phase flow model (3) in section 2.1 concerns the exact form of the right hand side, which reads now:

$$
R H S_{\eta}(\mathbf{W})=\nabla_{W} \eta(\mathbf{W}) \cdot \mathbf{r}(\mathbf{A}, \mathbf{W})
$$

or:

$$
R H S_{\eta}(\mathbf{W})=-\Sigma_{k=1}^{3} \Sigma_{l=1}^{3}\left(e_{k l}^{A}(A, \mathbf{W})\left|\mathbf{U}_{\mathbf{l}}-\mathbf{U}_{\mathbf{k}}\right|^{2}+d_{k l}(\mathbf{W})\left(P_{k}-P_{l}\right)^{2}\right) / 2
$$

\section{Remark 1:}

We may introduce $\psi_{A}=A / m_{1}$, and some positive function $h\left(\psi_{A}\right)$ such that $h^{\prime}\left(\psi_{A}\right) \leq 0$ and $0 \leq h^{\prime \prime}\left(\psi_{A}\right)$. We also note :

$$
\eta_{A}(A, \mathbf{W})=\eta(\mathbf{W})+m_{1} k_{0}^{2} h\left(\psi_{A}\right)
$$

with $k_{0} \in \mathscr{R}$, and :

$$
\mathbf{f}_{\eta, \mathbf{A}}(\mathbf{A}, \mathbf{W})=\mathbf{f}_{\eta}(\mathbf{W})+m_{1} k_{0}^{2} h\left(\psi_{A}\right) \mathbf{U}_{\mathbf{1}}
$$

Then regular solutions of (17) agree with

$$
\frac{\partial \eta_{A}(A, \mathbf{W})}{\partial t}+\nabla .\left(\mathbf{f}_{\eta, \mathbf{A}}(\mathbf{A}, \mathbf{W})\right) \leq 0
$$

Proof: starting from the balance equation for $A$ and using the mass balance equation for phase 1, we get:

$$
\frac{\partial \psi_{A}}{\partial t}+\mathbf{U}_{\mathbf{1}} \cdot \nabla \psi_{A}=g(A, \mathbf{W}) / m_{1}
$$

Thus, using the mass balance equation of phase 1 once more: 


$$
\frac{\partial m_{1} h\left(\psi_{A}\right)}{\partial t}+\nabla \cdot\left(m_{1} \mathbf{U}_{\mathbf{1}} h\left(\psi_{A}\right)\right)=g(A, \mathbf{W}) h^{\prime}\left(\psi_{A}\right)
$$

Meanwhile, we have:

$$
\frac{\partial \eta(\mathbf{W})}{\partial t}+\nabla \cdot\left(\mathbf{f}_{\eta}(\mathbf{W})\right)=\nabla_{W} \eta(\mathbf{W}) \cdot \mathbf{r}(\mathbf{A}, \mathbf{W}) \leq 0
$$

which, after summation, ends up with:

$$
\frac{\partial \eta_{A}(A, \mathbf{W})}{\partial t}+\nabla \cdot\left(\mathbf{f}_{\eta_{, \mathbf{A}}}(\mathbf{A}, \mathbf{W})\right)=\nabla_{W} \eta(\mathbf{W}) \cdot \mathbf{r}(\mathbf{A}, \mathbf{W})+k_{0}^{2} g(A, \mathbf{W}) h^{\prime}\left(\psi_{A}\right)
$$

This enables to conclude since $h^{\prime}\left(\psi_{A}\right) \leq 0$.

It is indeed useful to go back to the pure one-dimensional framework in order to examine some particularities of the modified three-phase flow model. Thus, if we focus on the pure $1 D$ model that writes:

$$
\left\{\begin{array}{l}
\frac{\partial m_{1} \psi_{A}}{\partial t}+\frac{\partial m_{1} u_{1} \psi_{A}}{\partial x}=0 \\
\frac{\partial \alpha_{k}}{\partial t}+u_{1} \frac{\partial \alpha_{k}}{\partial x}=0 \\
\frac{\partial m_{k}}{\partial t}+\frac{\partial m_{k} u_{k}}{\partial x}=0 \\
\frac{\partial m_{k} u_{k}}{\partial t}+\frac{\partial m_{k} u_{k}^{2}+\alpha_{k} P_{k}}{\partial x}+\Sigma_{l=1, l \neq k}^{3} \Pi_{k l}(\mathbf{W}) \frac{\partial \alpha_{l}}{\partial x}=0 .
\end{array}\right.
$$

We may introduce the non-conservative state vector $\mathbf{Y}$ such that:

$$
\mathbf{Y}^{T}=\left(\psi_{A}, \mathbf{X}^{\mathbf{T}}\right)
$$

Then, for regular solutions, the homogeneous convective part of system (24) reads:

$$
\left\{\begin{array}{l}
\frac{\partial \psi_{A}}{\partial t}+u_{1} \frac{\partial \psi_{A}}{\partial x}=0 \\
\frac{\partial \mathbf{X}}{\partial t}+\mathbf{C}(\mathbf{X}) \frac{\partial \mathbf{X}}{\partial x}=0
\end{array}\right.
$$

which may also be written in a more compact form:

$$
\frac{\partial \mathbf{Y}}{\partial t}+\mathscr{C}(\mathbf{Y}) \frac{\partial \mathbf{Y}}{\partial x}=0
$$

We get at once:

\section{Proposition 2:}


- The convective subset (24) admits nine real eigenvalues which read:

$$
\lambda_{0,1,2}(\mathbf{Y})=u_{1} \quad ; \quad \lambda_{3-8}(\mathbf{Y})=u_{k} \pm c_{k} .
$$

The field associated with eigenvalues $\lambda_{0,1,2}(\mathbf{Y})$ is linearly degenerate. Fields associated with $\lambda_{3-8}(\mathbf{Y})$ are genuinely non linear.

- $\psi_{A}$ is a Riemann inavariant within each GNL field.

- The structure of the coupling wave is unchanged, since the six Riemann invariants in the $0-1-2$ coupling wave write:

$$
\begin{gathered}
I_{0,1,2}^{1}(\mathbf{Y})=u_{1} \quad ; \quad I_{0,1,2}^{2}(\mathbf{Y})=m_{2}\left(u_{2}-u_{1}\right) \quad ; \quad I_{0,1,2}^{3}(\mathbf{Y})=m_{3}\left(u_{3}-u_{1}\right) ; \\
I_{0,1,2}^{4}(\mathbf{Y})=\frac{\left(u_{1}-u_{2}\right)^{2}}{2}+\int_{0}^{\rho_{2}}\left(\frac{c_{2}^{2}(x)}{x} d x\right) \quad ; \quad I_{0,1,2}^{5}(\mathbf{Y})=\frac{\left(u_{1}-u_{3}\right)^{2}}{2}+\int_{0}^{\rho_{3}}\left(\frac{c_{3}^{2}(x)}{x} d x\right) ; \\
I_{0,1,2}^{6}(\mathbf{Y})=\Sigma_{k=1}^{3}\left(\alpha_{k} P_{k}\right)+m_{2}\left(u_{2}-u_{1}\right)^{2}+m_{3}\left(u_{3}-u_{1}\right)^{2} .
\end{gathered}
$$

- If $\sigma$ denotes the speed of the discontinuity separating $\mathbf{Y}_{l}, \mathbf{Y}_{r}$ states in an isolated GNL wave, jump conditions between $\mathbf{Y}_{l}, \mathbf{Y}_{r}$ states are:

$$
\left\{\begin{array}{l}
{\left[\alpha_{k}\right]_{l}^{r}=\left[\psi_{A}\right]_{l}^{r}=0} \\
{\left[\rho_{k}\left(u_{k}-\sigma\right)\right]_{l}^{r}=0} \\
{\left[\rho_{k}\left(u_{k}-\sigma\right)^{2}+P_{k}\right]_{l}^{r}=0}
\end{array}\right.
$$

Proof: if $\mathbf{r}_{\mathbf{0}}(\mathbf{X}), \mathbf{r}_{\mathbf{1}}(\mathbf{X})$ denote the two right-eigenvectors associated with the double eigenvalue $\lambda_{0,1}(X)=u_{1}$ of matrix $\mathbf{C}(\mathbf{X})$, we define:

$$
\mathbf{R}_{\mathbf{0}}(\mathbf{Y})^{T}=\left(1, \mathbf{O}^{T}\right)
$$

and, for $k=1,2$ :

$$
\mathbf{R}_{\mathbf{k}}(\mathbf{Y})^{T}=\left(0, \mathbf{r}_{\mathbf{k}-\mathbf{1}}(\mathbf{X})^{T}\right)
$$

Now, we may compute, for $m=1-6$ :

$$
\nabla_{Y}\left(I_{0,1,2}^{m}(\mathbf{Y})\right) \cdot \mathbf{R}_{\mathbf{0}}(\mathbf{Y})=\left(0, \nabla_{X}\left(I_{0,1}^{m}(\mathbf{X})\right)^{T}\right) \cdot\left(1, \mathbf{O}^{T}\right)^{T}=0
$$

and, for $k=1,2$ :

$\left.\nabla_{Y}\left(I_{0,1,2}^{m}(\mathbf{Y})\right) \cdot \mathbf{R}_{\mathbf{k}}(\mathbf{Y})=\left(0, \nabla_{X}\left(I_{0,1,2}^{m}(\mathbf{Y})\right)^{T}\right)\left(0, \mathbf{r}_{\mathbf{k}-1}(\mathbf{X})^{T}\right)^{T}=\nabla_{X}\left(I_{0,1}^{m}(\mathbf{X})\right)\right) \cdot \mathbf{r}_{\mathbf{k}-\mathbf{1}}(\mathbf{X})=0$

owing to property 3. Evenmore, defining $\mathbf{R}_{\mathbf{k}}(\mathbf{Y})^{T}=\left(0, \mathbf{r}_{\mathbf{k}-\mathbf{1}}(\mathbf{X})^{T}\right)$, for $k=3-8$, where $\mathbf{r}_{\mathbf{k}-\mathbf{1}}(\mathbf{X})$ stands for the $(k-1)$-th right-eigenvector of $\mathbf{C}(\mathbf{X})$, we end up with: 


$$
\nabla_{Y}\left(\psi_{A}\right) \cdot \mathbf{R}_{\mathbf{k}}(\mathbf{Y})=\left(1, \mathbf{0}^{T}\right)\left(0, \mathbf{r}_{\mathbf{k}-\mathbf{1}}(\mathbf{X})^{T}\right)^{T}=0
$$

Furthermore, if we turn to jump conditions within an isolated GNL field, we have:

$$
\left\{\begin{array}{l}
-\sigma\left[m_{1} \psi_{A}\right]_{l}^{r}+\left[m_{1} \psi_{A} u_{1}\right]_{l}^{r}=0 \\
-\sigma\left[m_{1}\right]_{l}^{r}+\left[m_{1} u_{1}\right]_{l}^{r}=0
\end{array}\right.
$$

and thus:

$$
{\overline{m_{1}\left(u_{1}-\sigma\right)}}_{l r}\left[\psi_{A}\right]_{l}^{r}=0
$$

with the classical notation $\bar{\psi}_{l r}=\left(\psi_{l}+\psi_{r}\right) / 2$, or equivalently:

$$
m_{1}\left(u_{1}-\sigma\right)\left[\psi_{A}\right]_{l}^{r}=0
$$

\section{Remark 2:}

Using the above jump conditions in the GNL field associated with eigenvalue $u_{1} \pm c_{1}$ within phase 1 we note that:

$$
[A]_{l}^{r}=\left[m_{1} \psi_{A}\right]_{l}^{r}=\psi_{A}\left[m_{1}\right]_{l}^{r}=\psi_{A} \alpha_{1}\left[\rho_{1}\right]_{l}^{r}
$$

This means that for the weakly compressible liquid within phase 1 , we may expect slight variations of the interfacial area $A$ through phase 1 shock waves, though $\alpha_{1}$ does not vary through the latter field.

\section{Remark 3:}

The function $g(A, W)$ that will be used in practical computations will only take breakup phenomenum into account, and effects of coalescence will be neglected, which means that $A$ will always be non-decreasing. Of course other forms are available in the litterature, in order to account for coalescence (see [17, 31, 32, 37] among others, and also numerous references therein), and alternative closure laws for the breakup can also be found in the latter references. We emphasize that other convective patterns are also proposed in $[24,37]$ for the interfacial area $A$, which are not under conservative form. This of course would raise the problem of how to close jump conditions associated with $A$.

\subsection{Some comments on the pressure-velocity relaxation process}

We turn now to some aspects related to the pressure-velocity relaxation process involved in the latter three-phase flow models. For readers interested in theoretical aspects in relaxation processes occuring in two phase flow models, we refer for instance to [14]. Now, some specificities immediately arise for three-phase flows, which are mainly due to the fact that three one-to-one connections are present in 
the whole interfacial transfer. In [21], a few results were given when restricting to "isotropic" relaxation time scales. Hence the system of interest is :

$$
\left\{\begin{array}{l}
\frac{\partial \alpha_{k}}{\partial t}=\phi_{k}(\mathbf{W}) ; \\
\frac{\partial m_{k}}{\partial t}=0 ; \\
\frac{\partial m_{k} \mathbf{U}_{\mathbf{k}}}{\partial t}=m_{k} \mathrm{~S}_{\mathrm{k}}(\mathbf{W}) .
\end{array}\right.
$$

and we define the quantities: $a_{k}=\rho_{k} c_{k}^{2} / \alpha_{k}$. We also introduce:

$$
\Delta P_{21}=P_{2}-P_{1} \quad ; \quad \Delta P_{23}=P_{2}-P_{3}
$$

and:

$$
\mathbf{Y}_{\mathbf{p}}=\left(\Delta P_{21}, \Delta P_{23}\right)^{T}
$$

Owing to the fact that the partial mass remains constant, we get first :

$$
\frac{\partial P_{k}}{\partial t}=-a_{k} \frac{\partial \alpha_{k}}{\partial t}
$$

Thus it comes:

$$
\frac{\partial \mathbf{Y}_{\mathbf{p}}}{\partial t}=-\mathscr{U}_{\mathbf{a}} \frac{\partial\left(\alpha_{2}, \alpha_{3}\right)^{T}}{\partial t}
$$

The $2 \times 2$ matrix $\mathscr{U}_{\mathbf{a}}$ has real coefficients :

$$
\left(\mathscr{U}_{\mathbf{a}}\right)_{11}=a_{1}+a_{2} ; \quad\left(\mathscr{U}_{\mathbf{a}}\right)_{12}=a_{1} ; \quad\left(\mathscr{U}_{\mathbf{a}}\right)_{21}=a_{2} ; \quad\left(\mathscr{U}_{\mathbf{a}}\right)_{22}=-a_{3}
$$

Its determinant reads:

$$
\delta_{a}=-\left(a_{1} a_{3}+a_{2} a_{3}+a_{1} a_{2}\right)<0
$$

Now we may also get the evolution of $\alpha_{2}$ and $\alpha_{3}$, so that:

$$
\frac{\partial\left(\alpha_{2}, \alpha_{3}\right)^{T}}{\partial t}=\mathscr{U}_{\mathbf{b}} \mathbf{Y}_{\mathbf{p}}
$$

where the $2 \times 2$ matrix $\mathscr{U}_{\mathbf{b}}$ is such that :

$$
\left(\mathscr{U}_{\mathbf{b}}\right)_{11}=d_{12} ; \quad\left(\mathscr{U}_{\mathbf{b}}\right)_{12}=d_{23} ; \quad\left(\mathscr{U}_{\mathbf{b}}\right)_{21}=d_{13} ; \quad\left(\mathscr{U}_{\mathbf{b}}\right)_{22}=-d_{13}-d_{23}
$$

$\mathscr{U}_{\mathbf{b}}$ determinant is:

$$
\delta_{b}=-\left(d_{12} d_{13}+d_{12} d_{23}+d_{13} d_{23}\right)<0
$$

Hence we get:

$$
\frac{\partial \mathbf{Y}_{\mathbf{p}}}{\partial t}=-\mathscr{U}_{\mathbf{a}} \mathscr{U}_{\mathbf{b}} \mathbf{Y}_{\mathbf{p}}=-\mathscr{U} \mathbf{Y}_{\mathbf{p}}
$$


If $\mu_{1}, \mu_{2}$ denote the two eigenvalues of $\mathscr{U}$, straightforward calculations give:

$$
\left\{\begin{array}{l}
\mu_{1} \mu_{2}=\operatorname{det}(\mathscr{U})=\delta_{a} \delta_{b}>0 \\
\mu_{1}+\mu_{2}=\operatorname{trace}(\mathscr{U})=d_{12}\left(a_{1}+a_{2}\right)+d_{13}\left(a_{1}+a_{3}\right)+d_{23}\left(a_{2}+a_{3}\right)>0 .
\end{array}\right.
$$

If we note $\Delta_{\mathscr{U}}=(\operatorname{trace}(\mathscr{U}))^{2}-4 \operatorname{det}(\mathscr{U})$, we end up with:

$$
\mu_{1,2}=\left(\operatorname{trace}(\mathscr{U}) \pm\left(\Delta_{\mathscr{U}}\right)^{1 / 2}\right) / 2
$$

We may conclude now that, unlike in the two-phase flow framework, the decay in the pressure relaxation process is not necessarily uniform, since the sign of $\Delta_{\mathscr{U}}$ is unknown in the general case. This means in practice that some oscillations might arise in some regions. The algorithm used in the next section will take advantage of the present analysis. Eventually, we note that a formal integration yields:

$$
\mathbf{Y}_{\mathbf{p}}(\mathbf{t})=\exp \left(-\int_{O}^{t}(\mathscr{U})(t) d t\right) \mathbf{Y}_{\mathbf{p}}(\mathbf{0})
$$

Though not detailed here, the velocity relaxation process is quite similar to the pressure relaxation process, but it is indeed more straightforward. If :

$$
\mathbf{Y}_{\mathbf{u}}=\left(\Delta U_{21}, \Delta U_{23}\right)^{T}
$$

denotes the two independent relative velocities, setting :

$$
\Delta U_{21}=U_{2}-U_{1} \quad ; \quad \Delta U_{23}=U_{2}-U_{3}
$$

then the following holds:

$$
\frac{\partial \mathbf{Y}_{\mathbf{u}}}{\partial t}=-\mathscr{V} \mathbf{Y}_{\mathbf{u}}
$$

where the $2 \times 2$ matrix $\mathscr{V}$ is given by:

$$
\left\{\begin{array}{c}
(\mathscr{V})_{11}=E_{12}+E_{21}+E_{13} ; \quad(\mathscr{V})_{12}=E_{23}-E_{13} \\
(\mathscr{V})_{21}=E_{21}-E_{31} ; \quad(\mathscr{V})_{22}=E_{23}+E_{31}+E_{32}
\end{array}\right.
$$

where :

$$
\left\{\begin{array}{l}
m_{1} E_{12}=m_{2} E_{21}=e_{12} \\
m_{1} E_{13}=m_{3} E_{31}=e_{13} \\
m_{2} E_{23}=m_{3} E_{32}=e_{23}
\end{array}\right.
$$

The trace and the determinant of matrix $\mathscr{V}$ respectively read:

$$
\operatorname{trace}(\mathscr{V})=e_{12}\left(\frac{1}{m_{1}}+\frac{1}{m_{2}}\right)+e_{13}\left(\frac{1}{m_{1}}+\frac{1}{m_{3}}\right)+e_{23}\left(\frac{1}{m_{2}}+\frac{1}{m_{3}}\right)>0
$$

and: 


$$
\operatorname{det}(\mathscr{V})=\left(e_{12} e_{13}+e_{12} e_{23}+e_{13} e_{23}\right)\left(\frac{1}{m_{1} m_{2}}+\frac{1}{m_{1} m_{3}}+\frac{1}{m_{2} m_{3}}\right)>0
$$

Hence we have the same kind of behaviour: the velocity relaxation process may be monotone if: $\Delta_{\mathscr{V}}=(\operatorname{trace}(\mathscr{V}))^{2}-4 \operatorname{det}(\mathscr{V})$ is positive, whereas it is not when $\Delta_{\mathscr{V}}$ is negative.

The numerical method that will be used for computational applications is presented and discussed below. It is actually the straightforward counterpart of the one used in $[22,11]$ for two-phase flow models. 


\section{Numerical method}

Owing to the former results, the temptation is great to introduce a fractional step method in order to compute approximate solutions of (3). Actually this one will guarantee positive values of discrete statistical fractions and partial masses, given a classical constraint on the time step. Moreover, by construction, it will be in agreement with the inner pressure-velocity relaxation processes. We will explain at the end of the section how to account for the additional interfacial area $A$.

We consider a classical Finite Volume formulation. The computational domain is meshed using unstructured cells $\Omega_{i}$, the surface of which is noted $\omega_{i} ; S_{i j}$ stands for the length of the $i j$ interface. At each interface separating cells $\Omega_{i}$ and $\Omega_{j}$, we define the unit outward normal vector $n_{i j}$ pointing from cell $\Omega_{i}$ towards $\Omega_{j}$. We define $\Delta t_{n}$ the time step at time such that: $t_{n+1}=t_{n}+\Delta t_{n}$. More over, $V(i)$ will refer to the neighbouring cells of $\Omega_{i}$.

\subsection{Fractional step method}

The time scheme is the following:

- Step 1 : Evolution step:

For a given initial condition $\mathbf{W}_{i}^{n}$, compute an approximate solution of $\mathbf{W}$ at time $t_{n+1}^{-}$, namely $\mathbf{W}^{n+1,-}$, by solving:

$$
\left\{\begin{array}{l}
\frac{\partial \alpha_{k}}{\partial t}+\mathscr{Y}_{\mathbf{i}}(\mathbf{W}) \nabla \alpha_{k}=0 \\
\frac{\partial m_{k}}{\partial t}+\nabla \cdot\left(m_{k} \mathbf{U}_{\mathbf{k}}\right)=0 \\
\frac{\partial m_{k} \mathbf{U}_{\mathbf{k}}}{\partial t}+\nabla \cdot\left(m_{k} \mathbf{U}_{\mathbf{k}} \times \mathbf{U}_{\mathbf{k}}+\alpha_{k} P_{k} \mathbf{I d}\right)+\Sigma_{l=1, l \neq k}^{3} \Pi_{k l}(\mathbf{W}) \nabla \alpha_{l}=0
\end{array}\right.
$$

- Step 2 : Relaxation step:

Starting with $\mathbf{W}^{n+1,-}$, compute an approximation $\mathbf{W}^{n+1}$ of the solution of:

$$
\left\{\begin{array}{l}
\frac{\partial \alpha_{k}}{\partial t}=\phi_{k}(\mathbf{W}) \\
\frac{\partial m_{k}}{\partial t}=0 \\
\frac{\partial m_{k} \mathbf{U}_{\mathbf{k}}}{\partial t}=m_{k} \mathrm{~S}_{\mathbf{k}}(\mathbf{W})
\end{array}\right.
$$




\subsection{Computing the relaxation step}

\subsubsection{Pressure relaxation}

Within each cell $\Omega_{i}$, starting with $\mathbf{W}_{\mathbf{i}}^{*}$, we compute the following sequence:

- Initialize : $\mathbf{y}_{\mathbf{p}}^{\mathbf{n}}=\mathbf{Y}_{\mathbf{p}}\left(\mathbf{W}_{\mathbf{i}}^{*}\right)$, where $\mathbf{Y}_{\mathbf{p}}$ is defined in (31);

- Compute the exact solution $\mathbf{y}_{\mathbf{p}}^{\mathbf{n}+\mathbf{1}}$ of the linear ODE:

$$
\frac{\partial \mathbf{y}_{\mathbf{p}}}{\partial t}=-\mathscr{U}\left(\mathbf{W}_{\mathbf{i}}^{*}\right) \mathbf{y}_{\mathbf{p}}
$$

at time $t=\Delta t_{n}$, using the initial condition $\mathbf{y}_{\mathbf{p}}^{\mathbf{n}}$, where the $2 \times 2$ matrix $\mathscr{U}$ is defined in (32);

- Find the pressure $x=\left(P_{1}\right)_{i}^{n+1}$ solution of the scalar equation $g_{p}(x)=1$ :

$$
g_{p}(x)=\frac{\left(m_{1}\right)_{i}^{*}}{\rho_{1}(x)}+\frac{\left(m_{2}\right)_{i}^{*}}{\rho_{2}\left(x+(\Delta P)_{21}^{n+1}\right)}+\frac{\left(m_{3}\right)_{i}^{*}}{\rho_{3}\left(x+(\Delta P)_{21}^{n+1}-(\Delta P)_{23}^{n+1}\right)}
$$

- Update $\left(P_{2}\right)_{i}^{n+1}$ and $\left(P_{3}\right)_{i}^{n+1}$, setting:

$$
\left(P_{2}\right)_{i}^{n+1}=x+(\Delta P)_{21}^{n+1} ; \quad\left(P_{3}\right)_{i}^{n+1}=x+(\Delta P)_{21}^{n+1}-(\Delta P)_{23}^{n+1}
$$

- Update $\left(\alpha_{k}\right)_{i}^{n+1}$ for $: k \in 1,2,3$, setting:

$$
\left(\alpha_{k}\right)_{i}^{n+1}=\frac{\left(m_{k}\right)_{i}^{*}}{\rho_{k}\left(\left(P_{k}\right)_{i}^{n+1}\right)}
$$

We now have the following result:

\section{Proposition 3:}

- We assume that EOS comply with the constraints (2). Then there exists a unique solution $x$ of (41) in the admissible range.

- Statistical fractions $\left(\alpha_{k}\right)_{i}^{n+1}$ lie in [0,1], and partial masses remain positive.

Proof: we define $x_{\min }=\max \left(0,-(\Delta P)_{21}^{n+1},(\Delta P)_{23}^{n+1}-(\Delta P)_{21}^{n+1}\right)$. Since $c_{k}^{2}>0$, the function $g_{p}(x)$ is decreasing for $x \in\left[x_{\min },+\infty\right.$. Moreover :

$$
\lim _{x \rightarrow+\infty} g_{p}(x)=0, \quad \text { and }: \quad \lim _{x \rightarrow x_{\min }} g_{p}(x)=+\infty .
$$

Thus there exists a unique solution to $g_{p}(x)=1$. Evenmore, this equation perfectly matches the condition:

$$
\left(\alpha_{1}\right)_{i}^{n+1}+\left(\alpha_{2}\right)_{i}^{n+1}+\left(\alpha_{3}\right)_{i}^{n+1}=1
$$

since $\left(m_{k}\right)_{i}^{*}=\left(m_{k}\right)_{i}^{n+1}$. This completes the proof. 
By the way, we note that for long time behaviours, the solution $x$ to $g_{p}(x)=1$ will exactly coincide with the equilibrium pressure. We may now examine the velocity relaxation step.

We also note that another implicit scheme, which is based on the initial algorithm [22], might also be used (see appendix A).

\subsubsection{Velocity relaxation}

Again, in each cell $\Omega_{i}$, starting with $\mathbf{W}_{\mathbf{i}}^{*}$, we compute the sequence:

- Initialize : $\mathbf{y}_{\mathbf{u}}^{\mathbf{n}}=\mathbf{Y}_{\mathbf{u}}\left(\mathbf{W}_{\mathbf{i}}^{*}\right)$, where $\mathbf{Y}_{\mathbf{u}}$ is defined in (34);

- Compute the exact solution $\mathbf{y}_{\mathbf{u}}^{\mathbf{n}+\mathbf{1}}$ of the linear ODE:

$$
\frac{\partial \mathbf{y}_{\mathbf{u}}}{\partial t}=-\mathscr{V}\left(\mathbf{W}_{\mathbf{i}}^{*}\right) \mathbf{y}_{\mathbf{u}}
$$

at time $t=\Delta t_{n}$, using the initial condition $\mathbf{y}_{\mathbf{u}}^{\mathbf{n}}$, where the $2 \times 2$ matrix $\mathscr{V}$ is defined in (35);

- Compute: $\left(\mathbf{U}_{\mathbf{1}}\right)_{i}^{n+1}$ as follows:

$$
\left(\mathbf{U}_{1}\right)_{i}^{n+1}=\left(\mathbf{U}_{e q}\right)_{i}^{n+1}-\frac{\left(\left(m_{2}\right)_{i}^{*}+\left(m_{3}\right)_{i}^{*}\right)(\Delta \mathbf{U})_{21}^{n+1}-\left(m_{3}\right)_{i}^{*}(\Delta \mathbf{U})_{23}^{n+1}}{\left(m_{1}\right)_{i}^{*}+\left(m_{2}\right)_{i}^{*}+\left(m_{3}\right)_{i}^{*}}
$$

where $\left(\mathbf{U}_{e q}\right)_{i}^{n+1}$ denotes the equilibrium velocity:

$$
\left(\mathbf{U}_{e q}\right)_{i}^{n+1}=\frac{\left(m_{1}\right)_{i}^{*}\left(\mathbf{U}_{1}\right)_{i}^{*}+\left(m_{2}\right)_{i}^{*}\left(\mathbf{U}_{2}\right)_{i}^{*}+\left(m_{3}\right)_{i}^{*}\left(\mathbf{U}_{3}\right)_{i}^{*}}{\left(m_{1}\right)_{i}^{*}+\left(m_{2}\right)_{i}^{*}+\left(m_{3}\right)_{i}^{*}}
$$

- Update: $\left(\mathbf{U}_{2}\right)_{i}^{n+1}$ and $\left(\mathbf{U}_{3}\right)_{i}^{n+1}$, while setting:

$$
\left(\mathbf{U}_{2}\right)_{i}^{n+1}=\left(\mathbf{U}_{1}\right)_{i}^{n+1}+(\Delta \mathbf{U})_{21}^{n+1} ; \quad\left(\mathbf{U}_{3}\right)_{i}^{n+1}=\left(\mathbf{U}_{1}\right)_{i}^{n+1}+(\Delta \mathbf{U})_{21}^{n+1}-(\Delta \mathbf{U})_{23}^{n+1}
$$

Again, we note that for large time steps, the three updated velocities will tend towards $\left(\mathbf{U}_{e q}\right)_{i}^{n+1}$. By construction, this scheme preserves the conservation of the total momentum.

\subsection{Computing the evolution step}

The scheme that is used in the sequel in order to compute the evolution step is nothing but the Rusanov scheme [35]. Thus, at each interface $i j$ separating cells $\Omega_{i}$ and $\Omega_{j}$, we define numerical normal fluxes : 


$$
\left\{\begin{array}{l}
\mathscr{F}_{n}^{\alpha_{k}}\left(\mathbf{W}, \mathbf{n}_{\mathbf{i j}}\right)=0 \\
\mathscr{F}_{n}^{m_{k}}\left(\mathbf{W}, \mathbf{n}_{\mathbf{i j}}\right)=m_{k} \mathbf{U}_{\mathbf{k}} \cdot \mathbf{n}_{\mathbf{i j}} \\
\mathscr{F}_{\mathbf{n}} \mathbf{Q}_{\mathbf{k}}\left(\mathbf{W}, \mathbf{n}_{\mathbf{i j}}\right)=m_{k} \mathbf{U}_{\mathbf{k}} \cdot \mathbf{n}_{\mathbf{i j}} \mathbf{U}_{\mathbf{k}}+\alpha_{k} P_{k} \mathbf{n}_{\mathbf{i j}}
\end{array}\right.
$$

and:

$$
\left\{\begin{array}{l}
2 \mathscr{G}_{n}^{\alpha_{k}}\left(\mathbf{W}_{\mathbf{i}}, \mathbf{W}_{\mathbf{j}}, \mathbf{n}_{\mathbf{i j}}\right)=-r_{i j}\left(\left(\alpha_{k}\right)_{j}-\left(\alpha_{k}\right)_{i}\right) ; \\
2 \mathscr{G}_{n}^{m_{k}}\left(\mathbf{W}_{\mathbf{i}}, \mathbf{W}_{\mathbf{j}}, \mathbf{n}_{\mathbf{i j}}\right)=\mathscr{F}_{n}^{m_{k}}\left(\mathbf{W}_{\mathbf{i}}, \mathbf{n}_{\mathbf{i j}}\right)+\mathscr{F}_{n}^{m_{k}}\left(\mathbf{W}_{\mathbf{j}}, \mathbf{n}_{\mathbf{i j}}\right)-r_{i j}\left(\left(m_{k}\right)_{j}-\left(m_{k}\right)_{i}\right) ; \\
2 \mathscr{G}_{\mathbf{n}}^{\mathbf{Q}_{\mathbf{k}}}\left(\mathbf{W}_{\mathbf{i}}, \mathbf{W}_{\mathbf{j}}, \mathbf{n}_{\mathbf{i j}}\right)=\mathscr{F}_{\mathbf{n}}^{\mathbf{Q}_{\mathbf{k}}}\left(\mathbf{W}_{\mathbf{i}}, \mathbf{n}_{\mathbf{i j}}\right)+\mathscr{F}_{\mathbf{n}}^{\mathbf{Q}_{\mathbf{k}}}\left(\mathbf{W}_{\mathbf{j}}, \mathbf{n}_{\mathbf{i j}}\right)-r_{i j}\left(\left(m_{k} \mathbf{U}_{\mathbf{k}}\right)_{j}-\left(m_{k} \mathbf{U}_{\mathbf{k}}\right)_{i}\right)
\end{array}\right.
$$

where the quantity $r_{i j}$ is defined by:

$$
r_{i j}=\max _{k=1-3}\left(\left(\left|\mathbf{U}_{\mathbf{k}} \cdot \mathbf{n}_{\mathbf{i j}}\right|+c_{k}\right)_{i},\left(\left|\mathbf{U}_{\mathbf{k}} \cdot \mathbf{n}_{\mathbf{i j}}\right|+c_{k}\right)_{j}\right)
$$

Whatever $\phi$ is, we also use the standard notation:

$$
\bar{\phi}_{i j}=\left(\phi_{i}+\phi_{j}\right) / 2
$$

Hence the solution is updated through the evolution step computing:

$$
\left\{\begin{array}{c}
\omega_{i}\left(\left(\alpha_{k}\right)_{i}^{n+1,-}-\left(\alpha_{k}\right)_{i}^{n}\right)+\Delta t^{n}\left(\Sigma_{j \in V(i)} \mathscr{G}_{n}^{\alpha_{k}}\left(\mathbf{W}_{\mathbf{i}}^{\mathbf{n}}, \mathbf{W}_{\mathbf{j}}^{\mathbf{n}}, \mathbf{n}_{\mathbf{i j}}\right) S_{i j}\right) \\
+\Delta t^{n}\left(\mathbf{U}_{\mathbf{1}}\right)_{i}^{n} \cdot\left(\Sigma_{j \in V(i)}\left(\bar{\alpha}_{k}\right)_{i j}^{n} \mathbf{n}_{\mathbf{i j}} S_{i j}\right)=0 \\
\omega_{i}\left(\left(m_{k}\right)_{i}^{n+1,-}-\left(m_{k}\right)_{i}^{n}\right)+\Delta t^{n}\left(\Sigma_{j \in V(i)} \mathscr{G}_{n}^{m_{k}}\left(\mathbf{W}_{\mathbf{i}}^{\mathbf{n}}, \mathbf{W}_{\mathbf{j}}^{\mathbf{n}}, \mathbf{n}_{\mathbf{i j}}\right) S_{i j}\right)=0 \\
\omega_{i}\left(\left(m_{k} \mathbf{U}_{\mathbf{k}}\right)_{i}^{n+1,-}-\left(m_{k} \mathbf{U}_{\mathbf{k}}\right)_{i}^{n}\right)+\Delta t^{n}\left(\Sigma_{j \in V(i)} \mathscr{G}_{\mathbf{n}}^{\mathbf{Q}_{\mathbf{k}}}\left(\mathbf{W}_{\mathbf{i}}^{\mathbf{n}}, \mathbf{W}_{\mathbf{j}}^{\mathbf{n}}, \mathbf{n}_{\mathbf{i j}}\right) S_{i j}\right) \\
+\Delta t^{n} \Sigma_{l=1, l \neq k}^{3} \Pi_{k l}(\mathbf{W})_{i}^{n}\left(\Sigma_{j \in V(i)}\left(\bar{\alpha}_{l}\right)_{i j}^{n} \mathbf{n}_{\mathbf{i j}} S_{i j}\right)=0
\end{array}\right.
$$

and we have the expected result:

Proposition 4:

The evolution step guarantees positive values of partial masses and statistical fractions if the time step complies with the constraint:

$$
\Delta t^{n}\left(\Sigma_{j \in V(i)} r_{i j} S_{i j}\right) \leq 2 \omega_{i}
$$

Proof: it is classical and omitted. Actually, the condition (47) is necessary and sufficient in order to rewrite $\left(\alpha_{k}\right)_{i}^{n+1,-}$ (respectively $\left(m_{k}\right)_{i}^{n+1,-}$ ) as a convex combination of the $\left(\alpha_{k}\right)_{j}^{n}$ (respectively $\left(m_{k}\right)_{j}^{n}$ ) for $j \in V(i)$. 


\subsection{Computing the interfacial area}

We take the interfacial area into account in a very simple manner. Owing to the particular form of its governing equation:

$$
\frac{\partial A}{\partial t}+\nabla \cdot\left(A \mathbf{U}_{\mathbf{1}}\right)=g(A, \mathbf{W})
$$

we compute convective fluxes in the evolution step:

$$
\frac{\partial A}{\partial t}+\nabla \cdot\left(A \mathbf{U}_{\mathbf{1}}\right)=0
$$

and then update $A$ by computing approximate solutions of the ODE:

$$
\frac{\partial A}{\partial t}=g(A, \mathbf{W})
$$

Before going further on, we detail the form of $g(A, \mathbf{W})$, which reads:

$$
g(A, \mathbf{W})=C_{0} \frac{A^{2}}{6 \alpha_{1}}\left(\frac{\rho_{p}}{\rho_{1}}\right)^{1 / 2}\left\|U_{1}-U_{p}\right\| f(W e)
$$

where $p$ (resp. 1) stands for the index of the liquid water (resp. liquid metal droplets), $C_{0}=0.245, W e=\rho_{1}\left\|U_{1}-U_{p}\right\|^{2} d_{1} / \sigma$ denotes the Weber number, and the function $f(W e)$ is null unless:

$$
f(W e)=1 \quad \text { if: } \quad W e>W e_{c r i t}
$$

$d_{1}$ and $\sigma$ respectively denote the diameter of liquid metal droplets and the surface tension. In practice, the critical Weber number $W e_{c r i t}$ needs to be given ; the value $W e_{c r i t}=12$ will be used in the computations below.

Thus, if we define $\mathscr{F}_{n}^{A}\left(\mathbf{W}, \mathbf{n}_{\mathbf{i j}}\right)=A \mathbf{U}_{\mathbf{1}} \cdot \mathbf{n}_{\mathbf{i j}}$ and :

$$
2 \mathscr{G}_{n}^{A}\left(\mathbf{W}_{\mathbf{i}}, \mathbf{W}_{\mathbf{j}}, \mathbf{n}_{\mathbf{i j}}\right)=\mathscr{F}_{n}^{A}\left(\mathbf{W}_{\mathbf{i}}, \mathbf{n}_{\mathbf{i j}}\right)+\mathscr{F}_{n}^{A}\left(\mathbf{W}_{\mathbf{j}}, \mathbf{n}_{\mathbf{i j}}\right)-r_{i j}\left(A_{j}-A_{i}\right)
$$

the approximate solution is advanced in time as follows:

$$
\omega_{i}\left(A_{i}^{n+1,-}-A_{i}^{n}\right)+\Delta t^{n}\left(\Sigma_{j \in V(i)} \mathscr{G}_{n}^{A}\left(\mathbf{W}_{\mathbf{i}}^{\mathbf{n}}, \mathbf{W}_{\mathbf{j}}^{\mathbf{n}}, \mathbf{n}_{\mathbf{i j}}\right) S_{i j}\right)=0 .
$$

Then it is updated in order to account for the dislocation term $g(A, \mathbf{W})$. Starting with cell values $A_{i}^{n+1,-}$, we compute :

$$
A_{i}^{n+1}=A_{i}^{n+1,-} \exp \left(\Delta \operatorname{th}\left(A_{i}^{n+1,-}, \mathbf{W}^{n+1}\right)\right)
$$

where: $h(A, \mathbf{W})=C_{0} \frac{A}{6 \alpha_{1}}\left(\frac{\rho_{p}}{\rho_{1}}\right)^{1 / 2}\left\|U_{1}-U_{p}\right\| f(W e)$. 
Starting with positive values for the $A_{j}^{n}$ in all cells, then positive values of $A_{i}^{n+1}$ are guaranteed, using the above algorithm, as soon as the time step complies with (47). 


\section{Numerical Results}

In the first subsection, we will examine three different Riemann problems in order to verify the numerical schemes. A first test case will correspond to the computation of a pure $0-1-2$ coupling wave (contact discontinuity), thus using results of proposition 2; afterwards a second Riemann problem involving a shock wave in phase 1 and the $0-1-2$ coupling wave will be investigated. The last one will be a classical shock tube problem. We restrict here to the classical three-phase flow model (3).

The second subsection will focus on the experimental set-up proposed in [8]. As mentioned briefly in the introduction, a shock tube apparatus will generate a shock wave propagating towards the right hand side, which will hit a cloud of spherical particles and interact with it. Two situations will be examined: in the first one, particles in the bed will be assumed to be rigid, whereas in the second one these particles will be liquid droplets that will be sheared by the hinting gas shock wave, and break up; this will strongly modify the structure of the flow, and the computation will take advantage of the fact that the interfacial area is estimated by the modified threephase flow model introduced in section 2.4. Hence we compute here approximate solutions of system (16).

We use uniform meshes, and set for all cases: $C F L=1 / 2$, which in turn enables to define the time step $\Delta t_{n}$.

\subsection{Verification test cases based on Riemann problems}

We consider simple EOS such that:

$$
P_{k}\left(\rho_{k}\right)=P_{k}^{0}\left(\rho_{k}\right)^{\gamma_{k}}
$$

where we set for all cases : $P_{k}^{0}=1.10^{5}$. Several values of polytropic exponents $\gamma_{k}$ will be used. As usual, the initial discontinuity separating states $W_{L}$ and $W_{R}$ is located at $x=1 / 2$, and the one-dimensional computational domain is $[0,1]$.

We neglect here relaxation terms, thus setting: $d_{k l}(W)=0$ and $e_{k l}(W)=0$, whatever $k, l$ are.

\subsubsection{Pure coupling wave}

For the first two test cases, EOS are chosen such that:

$$
\gamma_{1}=3 / 2, \quad \gamma_{2}=2, \quad \gamma_{3}=5 / 2
$$


The left state is such that $\left(U_{k}\right)_{L}=k,\left(V_{k}\right)_{L}=0$ and:

$$
\begin{gathered}
\left(\alpha_{2}\right)_{L}=4 / 10 \quad ; \quad\left(\alpha_{3}\right)_{L}=1 / 2 ; \\
\left(\rho_{1}\right)_{L}=1 / 3 \quad ; \quad\left(\rho_{2}\right)_{L}=1 / 4 \quad ; \quad\left(\rho_{3}\right)_{L}=1 .
\end{gathered}
$$

The right state labeled $W_{r}$ is defined setting $\left(V_{k}\right)_{r}=0$, but also:

$$
\left(\alpha_{2}\right)_{r}=2 / 10 \quad ; \quad\left(\alpha_{3}\right)_{r}=3 / 10 ;
$$

and computing the six unknowns $\left(\rho_{k}\right)_{r},\left(U_{k}\right)_{r}$ in such a way that:

$$
I_{0,1}^{m}\left(W_{L}\right)=I_{0,1}^{m}\left(W_{r}\right)
$$

for $m=1 \rightarrow 6$ (see section 2.3 for details).

Thus we obtain:

$$
\left(\rho_{1}\right)_{r}=0.598903, \quad ; \quad\left(\rho_{2}\right)_{r}=0.249992, \quad ; \quad\left(\rho_{3}\right)_{r}=0.999986,
$$

and:

$$
\left(U_{1}\right)_{r}=1.0, \quad ; \quad\left(U_{2}\right)_{r}=3.000060, \quad ; \quad\left(U_{3}\right)_{r}=4.333381 .
$$

We may thus compute the $L^{1}$ norm for the six independent variables $I_{0,1}^{m}(W)$. The coarser and finer regular meshes contain 100 and 50000 cells respectively. We retrieve as expected the $1 / 2$ rate of convergence for all variables (see Figure 2). Approximate values of the invariant $I_{0,1}^{6}(W)$ can be observed in Figure 1, while considering various meshes.

\subsubsection{Phase 1 shock wave coupled with the coupling wave}

This second Riemann problem relies on similar initial conditions $W_{L}$ on the left hand side. However, the right state $W_{R}$ is now taken equal to $W_{r}$, except for the two components $\left(\rho_{1}\right)_{R}$ and $\left(U_{1}\right)_{R}$ which read now:

$$
\left(\rho_{1}\right)_{R}=0.19963439, \quad\left(u_{1}\right)_{R}=-352,54023
$$

The exact solution is composed of a right-going coupling contact wave travelling at speed $\left(u_{1}\right)_{L}$ behind a 1 -shock wave travelling at speed $\sigma_{1}$. More exactly we have:

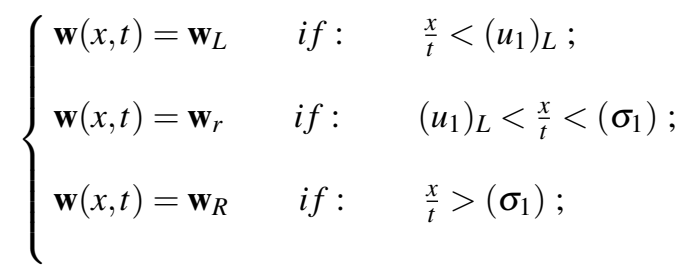




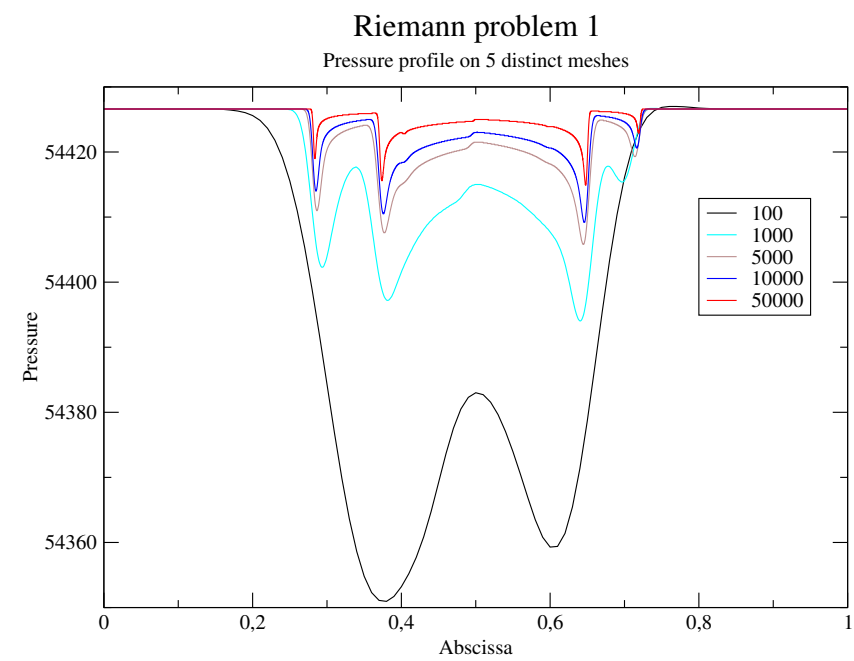

Fig. 1 Riemann problem 1. Pressure profiles for $I_{0,1}^{6}(W)$ on five distinct meshes. Coarser mesh: 100 cells, finer mesh : 50000 cells.

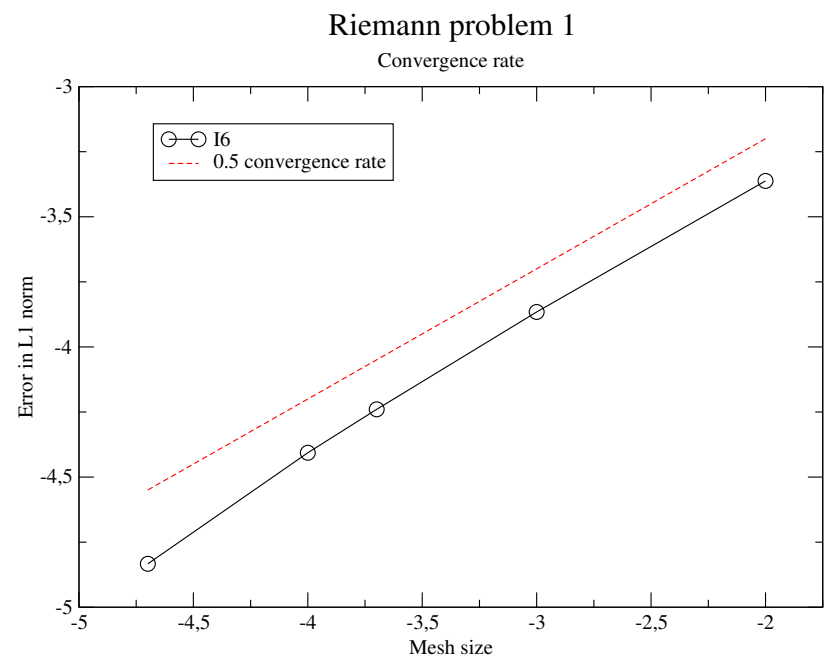

Fig. 2 Riemann problem 1. $L^{1}$ norm of the error for $I_{0,1}^{6}(W)$ wrt the mesh size $h$.

Using the same kind of meshes as before, we plot the $L^{1}$ norm of the error on figures 3 , 4. Again we retrieve a convergence behaviour as $h^{1 / 2}$ for fine enough meshes. The finer mesh here involves two hundred thousand cells. This hudge refinement is mandatory, owing to the competition between the asymptotic $h^{1 / 2}$ (respectively $h^{1}$ ) rates of convergence in the contact (respectively shock) waves. 


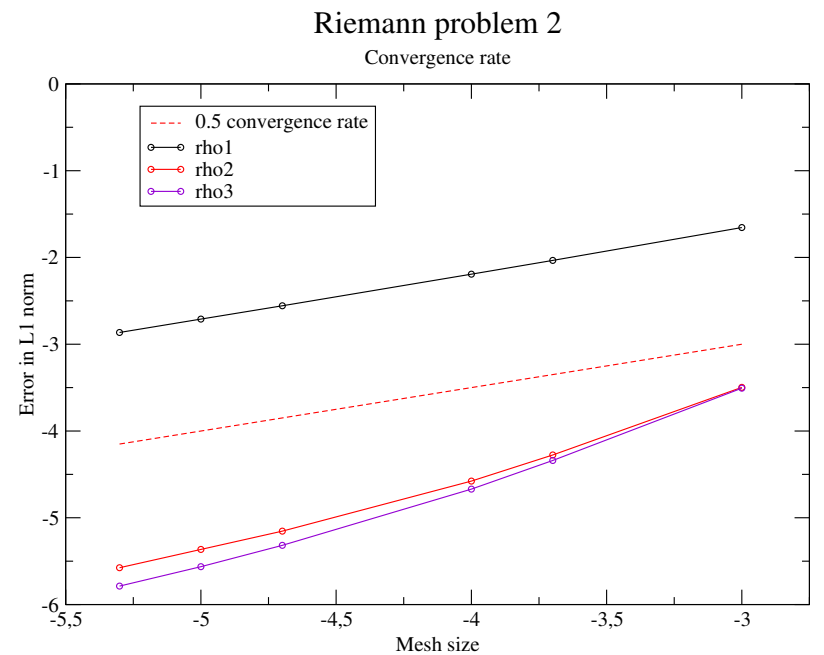

Fig. 3 Riemann problem 2. $L^{1}$ norm of the error (densities) wrt the mesh size $h$.

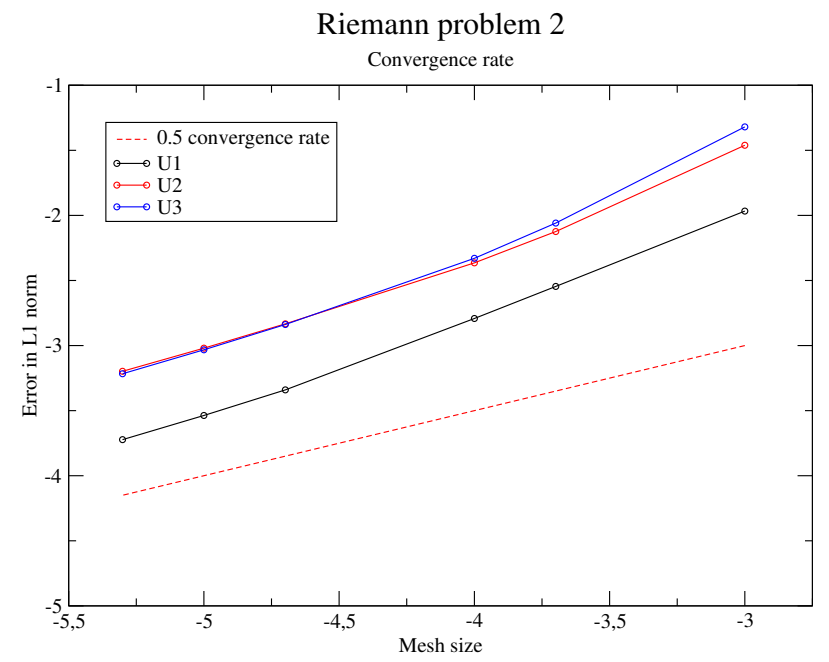

Fig. 4 Riemann problem 2. $L^{1}$ norm of the error (velocities) wrt the mesh size $h$.

\subsubsection{Pure shock tube problem}

Herein, EOS are such that:

$$
\gamma_{1}=7 / 5, \quad \gamma_{2}=1.005, \quad \gamma_{3}=1.001
$$




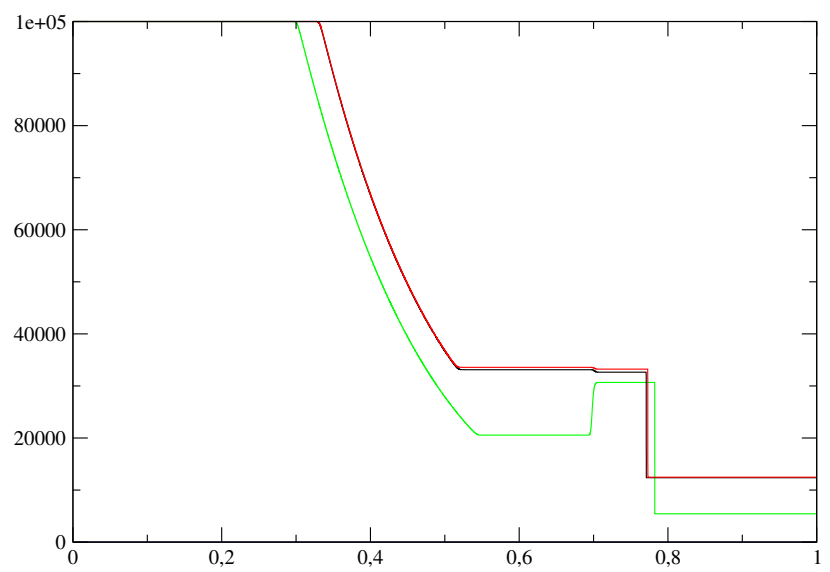

Fig. 5 Riemann problem 3. Pressure profiles on a fine mesh : $P_{1}$ (green), $P_{2}$ (black), $P_{3}$ (red).

We use here a fine mesh including 80000 regular cells. The initial data are such that velocities are null everywhere at the beginning of the computation, thus, for $k=1,2,3$ :

$$
\left(U_{k}\right)_{L}=\left(U_{k}\right)_{R}=\left(V_{k}\right)_{L}=\left(V_{k}\right)_{R}=0 ;
$$

In addition we choose left and right states $\left(\alpha_{k}\right)_{L, R}$ and $\left(\rho_{k}\right)_{L, R}$ such that:

$$
\begin{array}{cc}
\left(\alpha_{2}\right)_{L}=0.4 \quad ; \quad\left(\alpha_{3}\right)_{L}=0.5 \quad ; \quad\left(\alpha_{2}\right)_{R}=0.2 \quad ; \quad\left(\alpha_{3}\right)_{R}=0.3 ; \\
\left(\rho_{k}\right)_{L}=1 . \quad ; \quad\left(\rho_{k}\right)_{R}=1 / 8 .
\end{array}
$$

for $k=1$ to3. Phasic pressures $P_{k}$ have been plotted on Figure 5. Approximations of the sixth Riemann invariant: $\mathscr{P}=I_{0-1}^{6}(W)$ of the the $0-1$ coupling wave, and of the mean pressure:

$$
P_{\text {wall }}=\Sigma_{k=1 \rightarrow 3} \alpha_{k} P_{k}
$$

are given in Figure 6, while velocity profiles are drawn in Figure 7. We may check the numerical invariance of $\mathscr{P}=I_{0-1}^{6}(W)$ through the $0-1$ coupling wave.

\subsection{Shock tube apparatus: interaction of a gas shock wave with a lid of droplets}

The main objectives of this section are two-fold. We wish first to validate the numerical method built in order to provide approximate solutions of the barotropic three-phase flow model, with one vanishing phase (phase labeled 3 here). Moreover, we aim at investigating the two solutions associated with: 


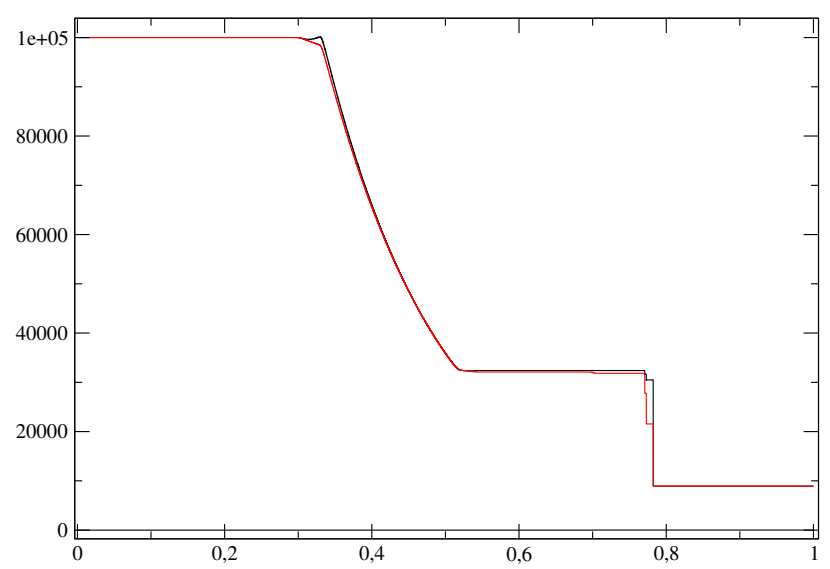

Fig. 6 Riemann problem 3. Pressure profiles on a fine mesh : $\mathscr{P}=I_{0,1}^{6}(W)$ (black), $P_{\text {wall }}$ (red).

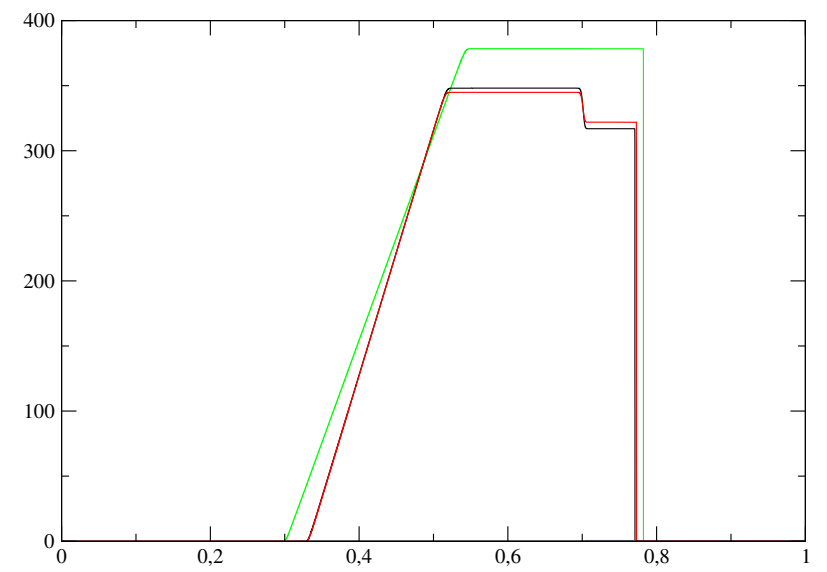

Fig. 7 Riemann problem 3. Velocity profiles on a fine mesh : $U_{1}$ (green), $U_{2}$ (black), $U_{3}$ (red).

1. the standard three-phase flow model (3),

2. the modified system (17) that accounts for the interfacial area $A$.

The first one will enable us to compute approximate solutions of an incoming gas shock wave hitting a cloud of spherical rigid particles, whereas the second one will generate similar approximations when a lid of deformable liquid droplets is considered instead.

For that purpose, we consider here the experimental setup discussed in [7, 8]. This one is indeed interesting and well documented. It is well suited for our basic goal: mechanical effects alone are at stake, which corresponds to our situation where the sole barotropic model is considered, rather than its full form [20] including par- 


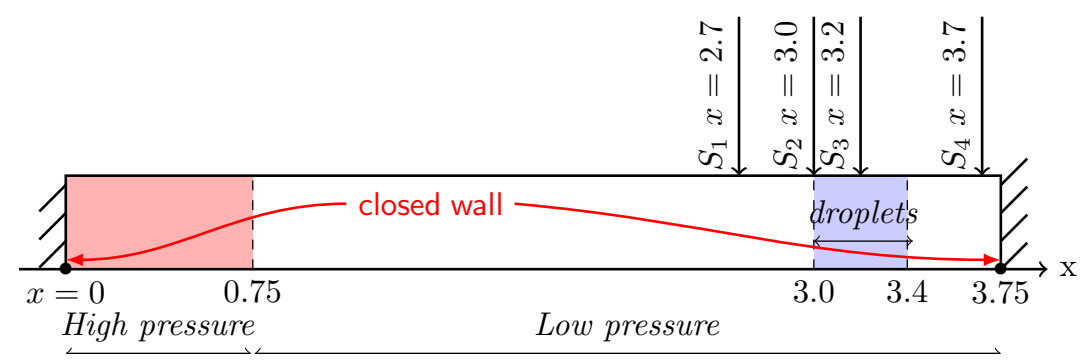

Fig. 8 Sketch of the experimental shock tube apparatus.

tial differential equations for the total energies. Hence we may expect to retrieve the main flow patterns pointed out in $[7,8]$; more precisely, pressure transducers reveal that, after the first instants of interaction of the incoming shock wave with the cloud of liquid droplets, a pressure decrease occurs until some new pressure equilibrium is retrieved; it thus clearly reduces the intensity of the shock wave.

A sketch of the shock tube apparatus is given in figure 8. Four pressure transducers are located at stations $S_{p}$ (for $p=1-4$ ). The length of the computational domain is $L=3.75 \mathrm{~m}$, and the tube is closed at both ends. The cloud of (rigid or liquid) droplets lies between $x_{1}=2.97 \mathrm{~m}$ and $x_{2}=3.37 \mathrm{~m}$ (hence the bed width is 400. $\mathrm{mm}$ ). The gas phase (with label 2) and the liquid-water droplet phase (with label 1) are at rest at the beginning of the computation, and all pressure fields are assumed to be in equilibrium with the gas phase everywhere, thus, for $k=1,3$ :

$$
P_{k}(x, t=0)=P_{2}(x, t=0) \text {. }
$$

The position of the initial pressure disequilibrium is $x_{0}=0.75$, and the mean gas pressure is given by ([7]) :

$$
P_{2}\left(x<x_{0}, t=0\right)=P_{L}=7 . \times 10^{5}, \quad P_{2}\left(x>x_{0}, t=0\right)=P_{R}=1 . \times 10^{5},
$$

on each side of the initial membrane.

The statistical fraction for the evanescent phase 3 is set to $\alpha_{3}=10^{-10}$ everywhere before the experiment starts.

Liquid water droplets in the cloud are spherical, identical and their initial diameter is $d(x, t=0)=0.5 \mathrm{~mm}$.

Eventually, the amount of droplets (or rigid droplets) within the lid is equal to :

$\alpha_{1}(x, t=0)=0.0104$ for $: \quad x \in\left[x_{1}, x_{2}\right], \quad \alpha_{1}(x, t=0)=10^{-10}$. elsewhere.

The EOS for the liquid water droplet phase 1 is chosen as follows: 


$$
P_{1}\left(\rho_{1}\right)=c_{1,0}^{2} \rho_{1}+P_{\text {ref }}
$$

where: $c_{1,0}=1500$, and $P_{r e f}$ is such that: $P_{1}\left(10^{3}\right)=10^{5}$, whereas for the gas phase we choose:

$$
P_{2}\left(\rho_{2}\right)=10^{5} \times\left(\rho_{2} / \rho_{2, \text { ref }}\right)^{7 / 5}, \quad \text { with }: \rho_{2, \text { ref }}=1.27 .
$$

The same EOS is chosen for the vanishing phase 3 .

The drag force between gas and liquid water droplets (or undeformable droplets) is obtained using the following closure law:

$$
e_{12}=0.125 \times A \rho_{2} C_{12}\left|u_{1}-u_{2}\right|
$$

where: $C_{12}=0.42$. Eventually we set in these computations : $d_{12}=10^{10}$. Unless otherwise specified, computations have been performed using regular meshes including $10^{4}, 5 . \times 10^{4}$ and $10^{5}$ cells, and the time step in chosen in order to comply with the $C F L=1 / 2$ constraint.

\section{Discussion}

\section{- Single phase reference experiment:}

As a reference case, we may first compute the single phase case of a pure gas shock tube apparatus, by removing the initial cloud of particles (hence setting the above value $\alpha_{1}=0.014$ to $\alpha_{1}=10^{-10}$ ). The total pressure is noted :

$$
P=\Sigma_{k=1}^{3}\left(\alpha_{k} P_{k}\right) .
$$

The pressure records at station 1 and station 4 during the first ten milliseconds are shown in figure 9 and 10. For this single phase case, we may define the mean pressure $P^{*}$ just behind the right going shock wave, and also the mean pressure $P^{* *}$ after the reflection of this shock wave on the right wall boundary. We may compute exact values $P^{*}, P^{* *}$, assuming that the gas phase is governed by:

1. the barotropic Euler equations, using notation $P^{*, \text { Eulerbaro }}, P^{* *, \text { Eulerbaro }}$,

2. the full Euler equations, using notation $P^{*, \text { Euler }}, P^{* *, \text { Euler }}$,

and we may compare these values with the experimental values $P^{*, e x p}, P^{* *, e x p}$. The latter two values can be found in $[7,8]$, which are approximately :

$$
P^{*, \exp }=2.4 \times 10^{5}, \quad P^{* *, \exp }=5.0 \times 10^{5} ;
$$

whereas exact values obtained with the (Euler and barotropic Euler) models are respectively: 


$$
P^{*, \text { Euler }}=2.46 \times 10^{5}, \quad P^{* *, \text { Euler }}=5.02 \times 10^{5} ;
$$

and:

$$
P^{* \text { Eulerbaro }}=2.78 \times 10^{5}, \quad P^{* *, \text { Eulerbaro }}=6.85 \times 10^{5} .
$$

Hence, we note that the full Euler equations provide a fair appoximation of the experimental results, whereas the barotropic Euler equations over estimate the latter. Obviously, the former exact values of $P^{*, \text { Eulerbaro }}, P^{* *, \text { Eulerbaro }}$ are retrieved by the three-phase barotropic code on sufficiently fine meshes (see figure $10)$, which was expected of course (and mandatory).

Looking at figure 10 , one can also note at station 4 that when $t>t_{1}=7.7 \mathrm{~ms}$, the second plateau value $P^{* *, \text { Eulerbaro }}$ is followed by a regular decrease, due to the reflection of the initial left-going 1-rarefaction wave that has reflected on the left wall boundary.

A straightforward consequence of the previous comparison in the single-phase framework is that we can hardly expect an accurate approximation of experimental results with the current barotropic three-phase flow model when turning to two-phase or three-phase flow experiments.

\section{- Two-phase experiment with rigid droplets:}

We turn now to the case of a lid of rigid droplets, setting $\alpha_{1}=0.0104$ in the range $\left[x_{1}, x_{2}\right]$ at the beginning of the computation.

Thus we compute approximate solutions of the three-phase flow model (3), getting rid of the interfacial area equation for $A$, and using the initial diameter $d(x, t)=d_{0}$ everywhere throughout the computation.

Hence, we focus now on figure 11. At station 1, the pressure first jumps up to the same value $P^{*, \text { Eulerbaro }}=2.78 \times 10^{5}$, and it then remains steady until the reflection of the left-going rarefaction wave meets that position (around time $t=5.91 \mathrm{~ms}$ ), as it occured in the single phase experiment (see figure 9). The latter plateau value is thus followed by a decrease of the pressure due the interaction with the reflection of the left going rarefaction wave, and then we note a second jump (around time $t=6.4 \mathrm{~ms}$ ), which is due to the reflection of the right-going gas shock wave that has hit the cloud of droplets, again followed by a smooth decrease of the pressure. The latter jump obviously cannot be observed on signals recorded at stations 2 and 3 since these are located on the right hand side of the bottom of the cloud.

If we turn to station 2 , we may observe the first jump of the pressure which is slightly lower than the value $P^{* \text { Eulerbaro }}=2.78 \times 10^{5}$. Once the gas has penetrated the cloud of droplets, the pressure smoothly increases (the lid of solid particles acts as a convergent nozzle) until $t=6.4 \mathrm{~ms}$. This is again followed by 
a decrease of the pressure, owing to the reflected rarefaction wave, and again by a sudden compression due to the reflection of the initial right going shock wave on the right wall boundary.

Similar comments hold for the pressure signal at station 3, with a small delay, as shown on figure 11.

\section{- Two-phase experiment with deformable liquid droplets:}

The last test corresponds to the interaction of the gas shock wave with a lid of deformable liquid droplets. Hence we compute now approximate solutions of the modified three-phase flow model (17), including the governing equation for the interfacial area $A$.

Figures 12 and 13 provide numerical approximations of the mean pressure $P=\Sigma_{k=1}^{3}\left(\alpha_{k} P_{k}\right)$ obtained with the code at stations 2 and 3 in that case ; meanwhile figures 14 and 15 respectively show the behaviour of the interfacial area $A$ and of the void fraction $\alpha_{1}$ within the liquid phase.

At the beginning, we still note the same kind of flow pattern, and we retrieve a value of the mean pressure which is close to $P^{*, \text { Eulerbaro }}$. Nonetheless, and unlike in the previous case involving rigid droplets, it must emphasized that the mean pressure suddenly decreases just after the impact of the incoming gas shock wave with the lid of droplets. This behaviour is typical of what happens in a medium involving deformable droplets with a high breakup phenomenon (see [17]). This is actually well detailed in reference [8]. The present numerical simulation enables us to retrieve this specific behaviour, and this is indeed a very encouraging point. Afterwards, we note that the pressure $P$ increases again until it reaches some maximum value close to $4.8 \times 10^{5}$ (respectively $3.1 \times 10^{5}$ ) at station 2 (respectively at station 3 ). The experimental counterparts arising from $[7,8]$ for the latter two values are respectively $3.2 \times 10^{5}$ and $3.25 \times 10^{5}$.

Thus, as expected within the light of our preliminary single phase flow computations, the barotropic model provides a rather good qualitative agreement with experiment, and it enables to retrieve the sudden experimental decrease of the pressure in the lid of droplets once the transmitted incoming shock wave has hit the cloud, as soon as the breakup of droplets is accounted for in the model. However, the whole obviously requires further investigation and improvements in order to achieve a better matching with experimental results. Eventually, figure 16 shows the influence of the drag coefficient on the computations, hence comparing results associated to $C_{12}=0.42$ with those obtained with:

$$
C_{12}=\frac{24}{R e}\left(1+0.15(R e)^{0.687}\right)+0.42\left(1+42500(R e)^{-1.16}\right)^{-1}
$$

More details on this test case and associated results can be found in [6]. 
Station 1 pressure history

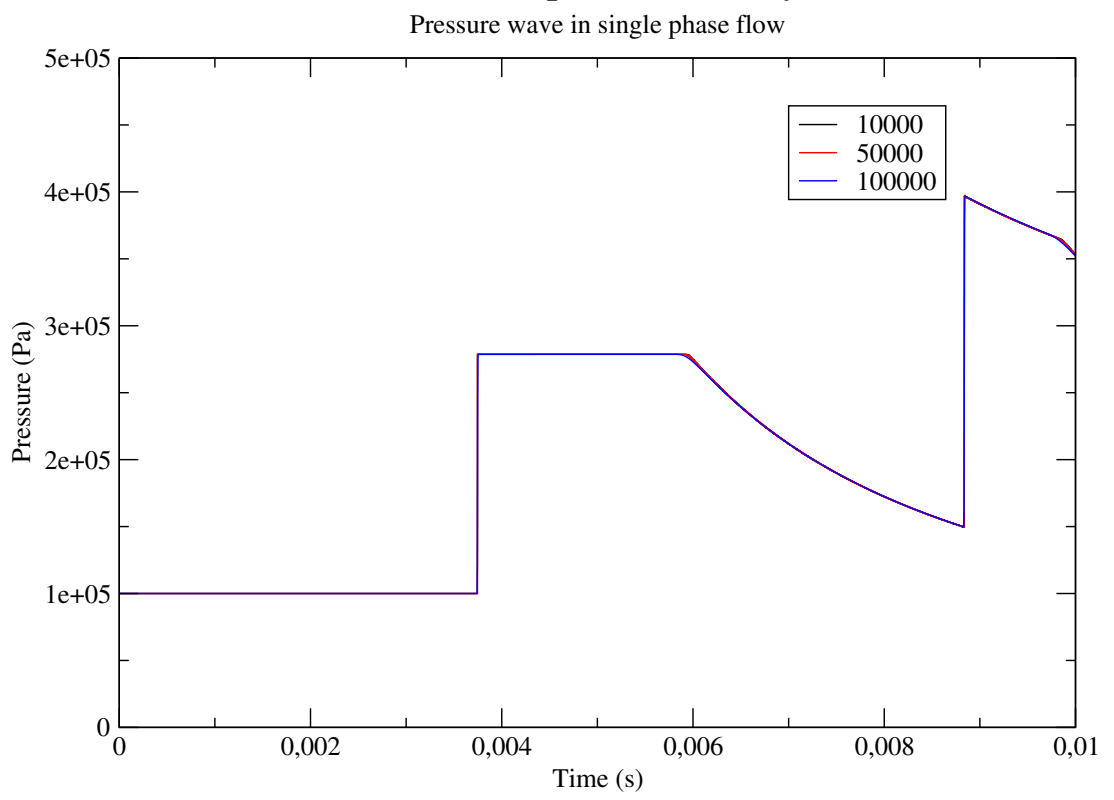

Fig. 9 Single phase shock tube apparatus. Pressure signal at station 1. Meshes : $10^{4}$ cells (black), 5. $\times 10^{4}$ cells (red) and $10^{5}$ cells (blue).

\section{Conclusion}

We have discussed in this paper the main properties of a three-phase flow barotropic model, and we gave special focus on the pressure and velocity relaxation process involved in the source terms. The fractional step method enables to comply with the entropy inequality at the continuous level, and it provides satisfactory results on fine meshes, as expected. It would be worth investigating alternative schemes in the pressure relaxation step in order to get a fully discrete entropy inequality. The comparison with the results of the experimental set-up of [8] is indeed encouraging. Applications to different situations such as those encountered in solid-gas-liquid flows in pipelines also seem quite appealing.

However, this work must also be understood as a first step in the direction of steam explosion modeling. Though the list below is not exhaustive, we detail afterwards some items that are still missing in order to obtain a complete and accurate enough approximation of that kind of flow.

Actually the following points immediately arise: 
Station 4 pressure history

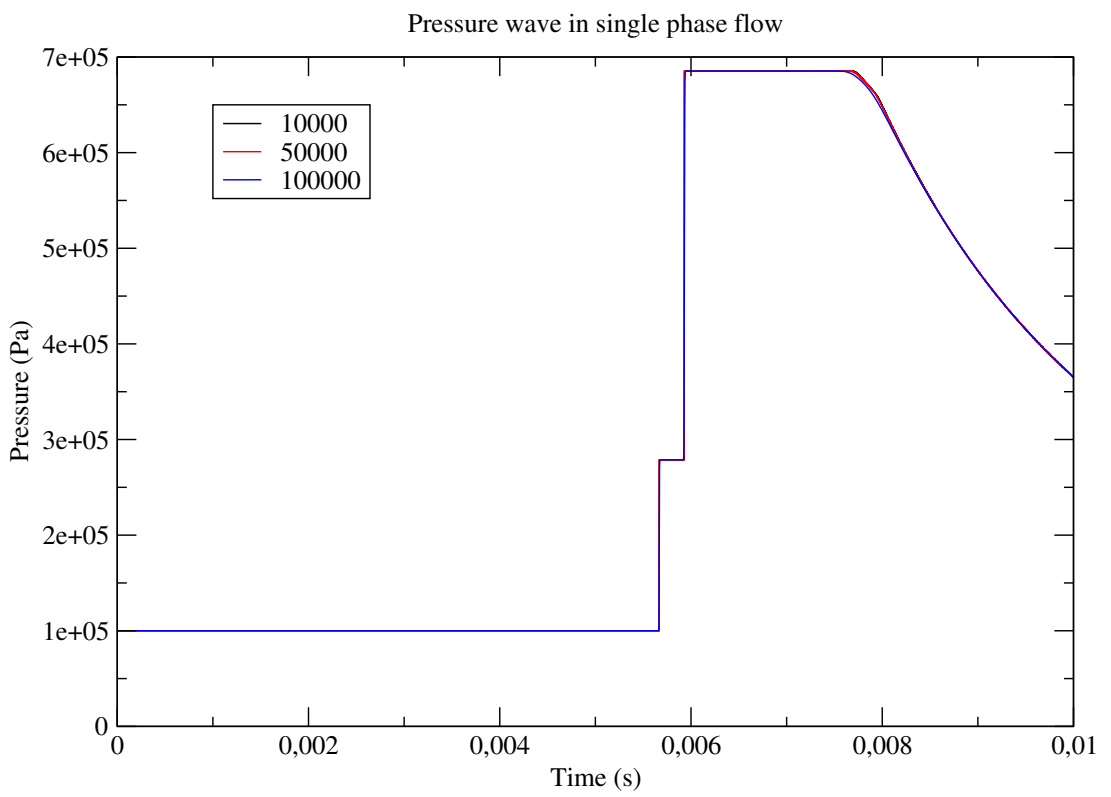

Fig. 10 Single phase shock tube apparatus. Pressure signal at station 4. Meshes : $10^{4}$ cells (black), 5. $\times 10^{4}$ cells (red)and $10^{5}$ cells (blue)

- First of all, the present work suggests that reliable and accurate enough $2 D$ or evenmore $3 D$ simulations will urge the development of approximate Riemann solvers dedicated to three phase flow models. An extension of the relaxation solvers introduced in $[1,2,11,10]$ seems promising for that purpose (see [36]);

- As underlined before, the barotropic approach provides a rather fair qualitative agreement with experimental results, but one obvious way to improve quantitative results requires the introduction of energy balance equations within each phase. Actually, the non-barotropic models provided in [20,29] are two possible candidates in order to take heat transfer into account, while being ensured that the barotropic part lies in the same hierarchy of models. One should nonetheless be aware that pressure relaxation processes are even more complex than those involved in the current barotropic model;

- A comparison of results obtained with the present model with extensions of the homogeneous models $[13,18,19]$ (involving a sole velocity field) would be worth being achieved;

- Eventually, the modification of the classical framework, which is due to the introduction of interfacial area equation, might certainly be improved. Possible ways in that direction are implicitly suggested in papers [27, 12, 24, 37], among others. 
Pressure history in stations 1 - 2 - 3

Pressure wave in two-phase gas-solid medium

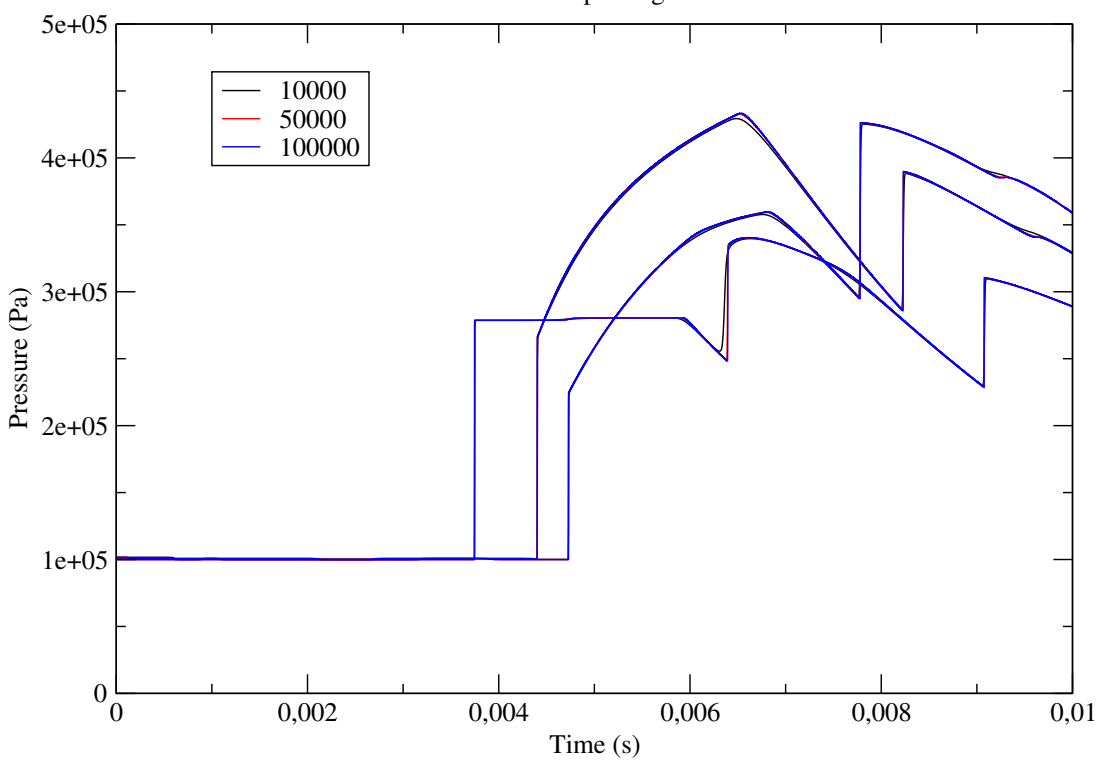

Fig. 11 Two-phase shock tube apparatus with solid particles. Mean pressure signal at stations $1-2-3$. Meshes : $10^{4}$ cells (black), $5 . \times 10^{4}$ cells (red)and $10^{5}$ cells (blue)

Acknowledgements The first author receives financial support by ANRT and EDF through an EDF/CIFRE grant number 2016/0611. Computational facilities were provided by EDF. Both authors would like to thank Thierry Gallouët, Sergey Gavrilyuk, Olivier Hurisse, Georges Jourdan, Jean-Claude Latché, Khaled Saleh and Nicolas Seguin for their help and for various useful discussions on the subject.

\section{Appendix A: an alternative pressure relaxation scheme}

An implicit Euler scheme might be used to compute pressures and statistical fractions in the pressure relaxation step, thus seeking solutions of:

$$
\left\{\begin{array}{l}
\left(\alpha_{2}\right)_{i}^{n+1}-\left(\alpha_{2}\right)_{i}^{*}=\Delta t^{n}\left(\Sigma_{k}\left(d_{2 k}\right)\left(P_{2}\left(\frac{m_{2}^{*}}{\alpha_{2}^{n+1}}\right)-P_{k}\left(\frac{m_{k}^{*}}{\alpha_{k}^{n+1}}\right)\right)\right) \\
\left(\alpha_{3}\right)_{i}^{n+1}-\left(\alpha_{3}\right)_{i}^{*}=\Delta t^{n}\left(\Sigma_{k}\left(d_{3 k}\right)\left(P_{3}\left(\frac{m_{3}^{*}}{\alpha_{3}^{n+1}}\right)-P_{k}\left(\frac{m_{k}^{*}}{\alpha_{k}^{n+1}}\right)\right)\right)
\end{array}\right.
$$

where of course : $\left(\alpha_{1}\right)_{i}^{n+1, *}=1-\left(\alpha_{2}\right)_{i}^{n+1, *}-\left(\alpha_{3}\right)_{i}^{n+1, *}$, with some abuse of notation. 
Station 2 pressure history

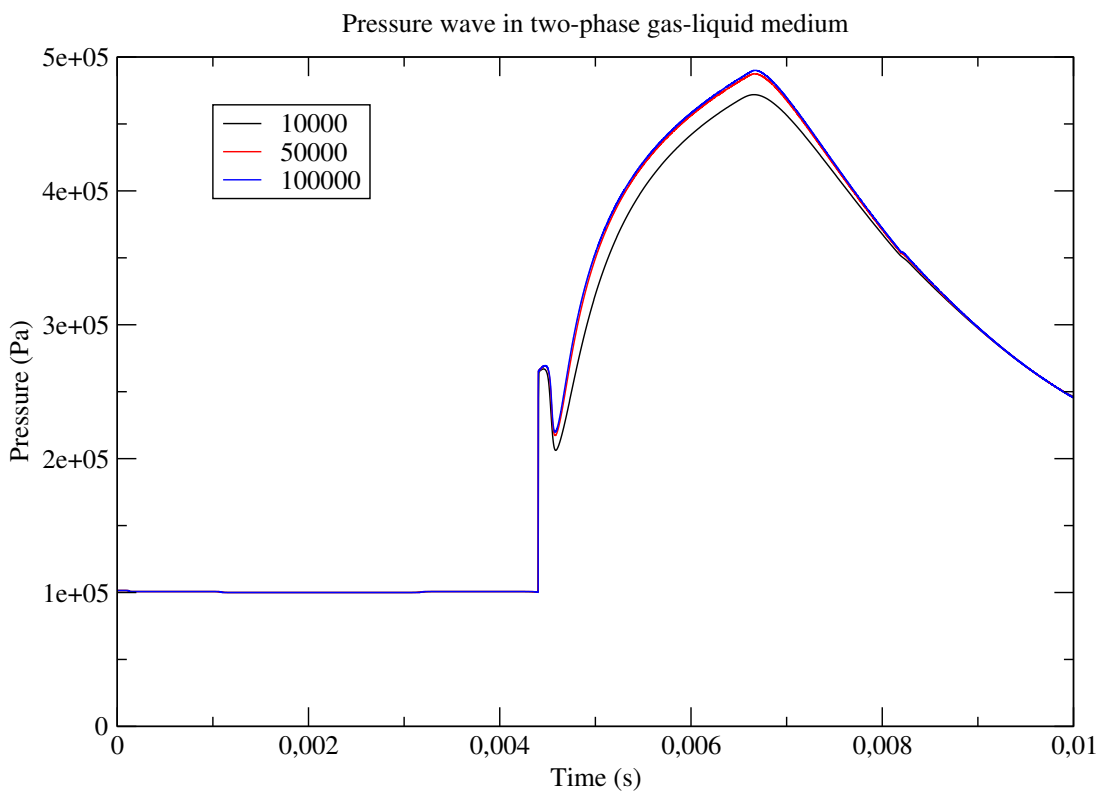

Fig. 12 Two-phase shock tube apparatus with liquid water droplets. Mean pressure signal at station 2. Meshes : $10^{4}$ cells (black), $5 . \times 10^{4}$ cells (red)and $10^{5}$ cells (blue)

Hence we have the result:

\section{Property:}

Assume that $\left(\alpha_{k}\right)_{i}^{*}$ and $\left(m_{k}\right)_{i}^{*}$ are positive. Then the previous discrete pressure relaxation scheme (49) computes a unique set of positive values $\left(\alpha_{k}\right)_{i}^{n+1}$.

One drawback of the latter scheme is that the discrete solution may be hard to find in practice, especially when statistical fractions are close to the boundary of the admissible domain ; some cure should be found in order to use the latter algorithm for practical computations. We underline that the latter scheme is the straightforward extension of the one used for two-phase flow models in [22].

\section{References}

1. A. Ambroso , C. Chalons, F. Coquel and T. Galié, Relaxation and numerical approximation of a two-fluid two-pressure diphasic model, ESAIM: Mathematical Modelling and Numerical Analysis, vol. 43, pp. 1063-1097, 2009.

2. A. Ambroso , C. Chalons AND P.A.Raviart, A Godunov type method for the sevenequation model of compressible two-phase flow, Computers and Fluids, vol. 54 , pp. 67-91, 
Station 3 pressure history

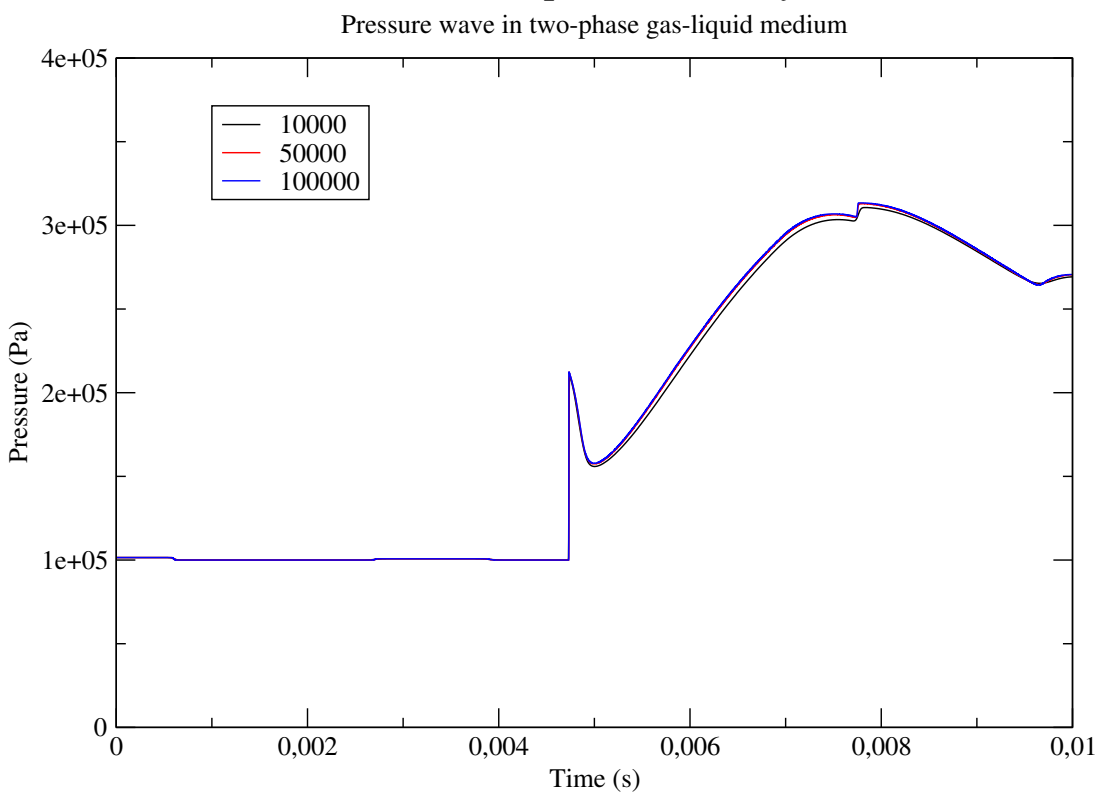

Fig. 13 Two-phase shock tube apparatus with liquid water droplets. Mean pressure signal at station 3. Meshes : $10^{4}$ cells (black), $5 . \times 10^{4}$ cells (red)and $10^{5}$ cells (blue)

2012.

3. M.R. BAer AND J.W. Nunziato, A two phase mixture theory for the deflagration to detonation transition (DDT) in reactive granular materials, Int. J. Multiphase Flow, vol. 12-6, pp. 861-889, 1986.

4. G. BerThOUd, Vapor explosions, Annual Review of Fluid Mechanics, vol. 32, pp. 573-611, 2000.

5. W. Bo, H. Jin, D. Kim, X. LiU, H. Lee, N. Pestiau, Y. Yu, J. Glimm and J.W. Grove, Comparison and validation of multiphase closure models, Computers and Mathematics with Applications, vol. 56, pp. 1291-1302, 2008.

6. H. Boukili, PhD thesis, in preparation, Université Aix Marseille.

7. A. Chauvin, Etude expérimentale de l'atténuation d'une onde de choc par un nuage de gouttes et validation numérique, $P h D$ thesis, Université Aix Marseille, 2012.

8. A. Chauvin, G. Jourdan, E. Daniel, L. Houas And R. Tosello, Experimental investigation of the propagation of a planar shock wave through a two-phase gas-liquid medium, Physcis of Fluids, vol.23,113301, 2011.

9. F. Coquel, T. Gallouët, J.M. Hérard and N. Seguin, Closure laws for a two fluid two-pressure model, C. R. Acad. Sci. Paris, vol. I-332, pp. 927-932, 2002.

10. F. Coquel, J.M. Hérard, K. SAleh and N. SEguin, A robust entropy-satisfying finite volume scheme for the isentropic Baer Nunziato model, ESAIM: Mathematical Modelling and Numerical Analysis, vol. 48, pp. 165-206, 2014.

11. F. COQuel, J.M. HÉRARD AND K. SALEH, A positive and entropy-satisfying finite volume scheme for the Baer Nunziato model, Journal of Computational Physics, vol. 330, pp. 401435, 2017.

12. M. ESSADKI, Contribution to a unified modelling of fuel injection: from dense liquid to polydisperse evaporating spray, $P h D$ thesis, Ecole polytechnique, february 13, 2018. 


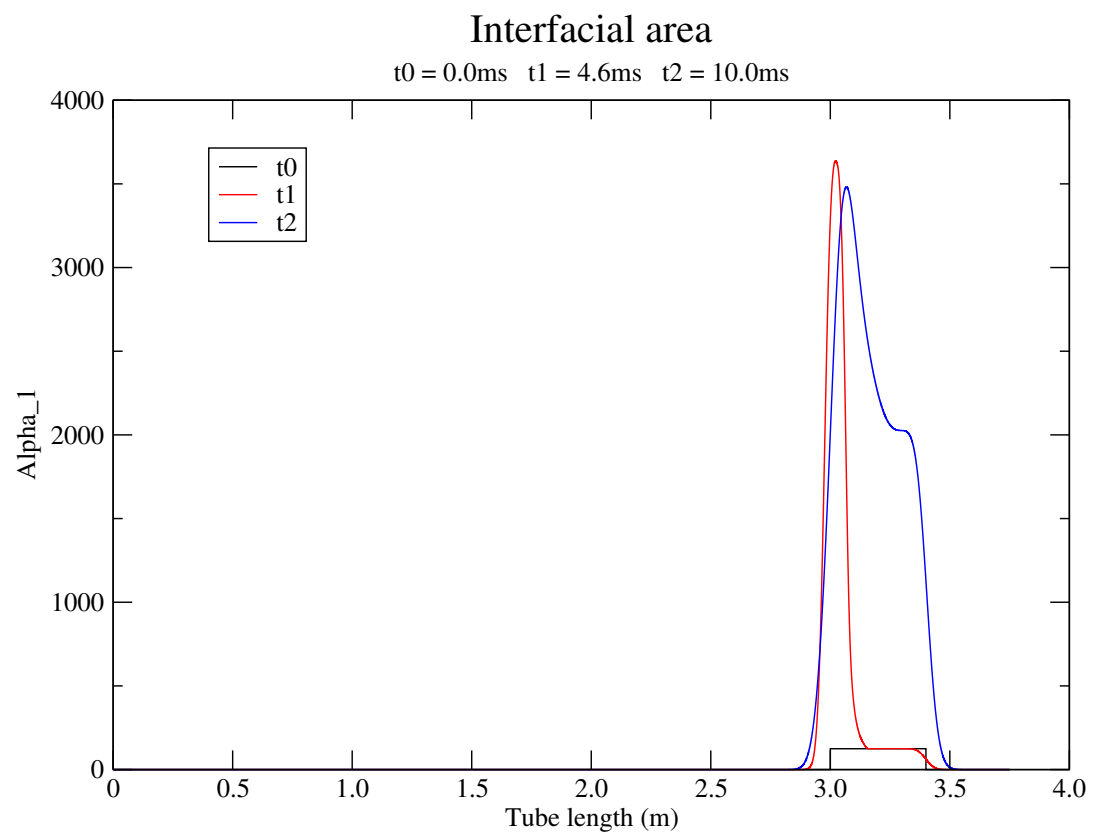

Fig. 14 Two-phase shock tube apparatus with liquid water droplets. Liquid water interfacial aerea profiles at three distinct instants.

13. G. FACCANONI, S. KoKh AND G. Allaire, Modelling and simulation of liquid-vapor phase transition in compressible flows based on thermodynamical equilibrium, ESAIM: Mathematical Modelling and Numerical Analysis, vol. 46, pp.1029-1054, 2012.

14. T. FLÄTtEN AND H. LUND, Relaxation two-phase flow models and the subcharacteristic condition. Mathematical Models and Methods in Applied Sciences, vol. 21(12), 2011.

15. S. GAVRILYUK, The structure of pressure relaxation terms: the one-velocity case, EDF report H-I83-2014-0276-EN, 2014

16. S. GaVRILYUK AND R. SAURel, Mathematical and numerical modelling of two-phase compressible flows with micro inertia, Journal of Computational Physics, vol. 175, pp. 326-360, 2002 .

17. B.E Gelfand, Droplet breakup phenomena in flows with velocity lag, Progress in Energy and Combustion Science, vol.22, pp.201-265, 1996.

18. P. Helluy AND H. MATHIS, Pressure laws and fast Legendre transform, Mathematical Models and Methods in Applied Sciences, vol. 21, pp.745-775, 2011.

19. P. Helluy and N. SEguin, Relaxation models of phase transition flows, ESAIM: Mathematical Modelling and Numerical Analysis, vol. 40, pp.331-352, 2006.

20. J.M. HÉRARD, A three-phase flow model, Mathematical and Computer Modelling, vol. 45, pp. 732-755, 2007.

21. J.M. HÉRARD, A class of compressible multiphase flow models, Comptes Rendus Mathématique, vol. 354, pp. 954-959, 2016.

22. J.M. HÉRARD AND O. HURISSE, A fractional step method to compute a class of compressible gas-liquid flows, Computers and Fluids, vol. 55, pp.57-69, 2012.

23. J.M. HÉrARd, K. SAleh AND N. SEguin, Some mathematical properties of a hyperbolic multiphase flow model, HAL preprint available on: https://hal.archives-ouvertes.fr/hal$01921027 v 1,2018$. 


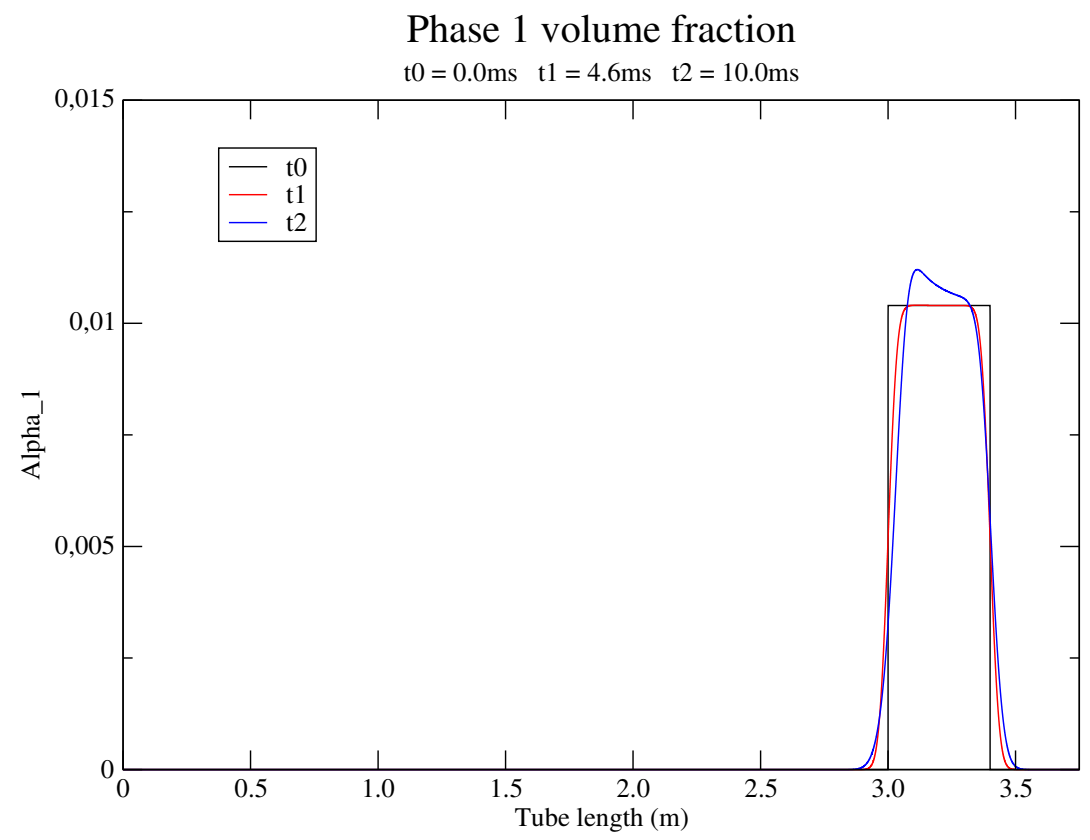

Fig. 15 Two-phase shock tube apparatus with liquid water droplets. Liquid water statistical fraction profiles at three distinct instants.

24. T. HibIKI AND M. IsHII, One-group interfacial area transport of bubbly flows in vertical round tubes, Int. J. of Heat and Mass Transfer, vol. 43, pp. 2711-2726, 2000.

25. A.K. Kapila, S.F. Son, J.B. Bdzil, R. Menikoff and D.S. STEwart, Two phase flow modeling of deflagration to detonation transition : srtucture of the velocity relaxation zone, Physics of Fluids, vol. 9(12), 1997.

26. H. MATHIS, A thermodynamically consistent model of a liquid-vapor fluid with a gas, ESAIM: Mathematical Modelling and Numerical Analysis, HAL preprint available on https://hal.archives-ouvertes.fr/hal-01615591v2, 2018.

27. M. Massot, F. Laurent, D. Kah and S. De Chaisemartin, A robust moment method for evaluation of the disappearance rate of evaporating sprays, SIAM Journal of Applied Mathematics, vol. 70, pp.3203-3234, 2010.

28. R. Meignen, B. Raverdy, S. Picchi and J. Lamome, The challenge of modelling fuelcoolant interaction. Part II: steam explosion, Nuclear Engineering and Design, vol. 280, pp.528-541, 2014.

29. S. MÜller, M. HANTKE AND P. RIChter, Closure conditions for non-equilibrium multicomponent models, Continuum Mechanics and Thermodynamics, vol. 28, pp. 1157-1190, 2016.

30. S. PICCHI, MC3D version 3.9. Description of the physical models of the premixing application, IRSN internal report PSN-RES/SAG/2017-0073, 2017.

31. M. PILCH, Acceleration induced fragmentation of liquid drops, $P h D$ thesis, University of Virginia, 1981.

32. M. PILCH AND C.A. ERDMAN, Use of breakup time data and velocity history data to predict the maximum size of stable fragments for acceleration induced breakup of a liquid drop, Int. J. Multiphase Flow, vol. 28, pp. 741-757, 1987. 
Station 2-3-4 pressure history

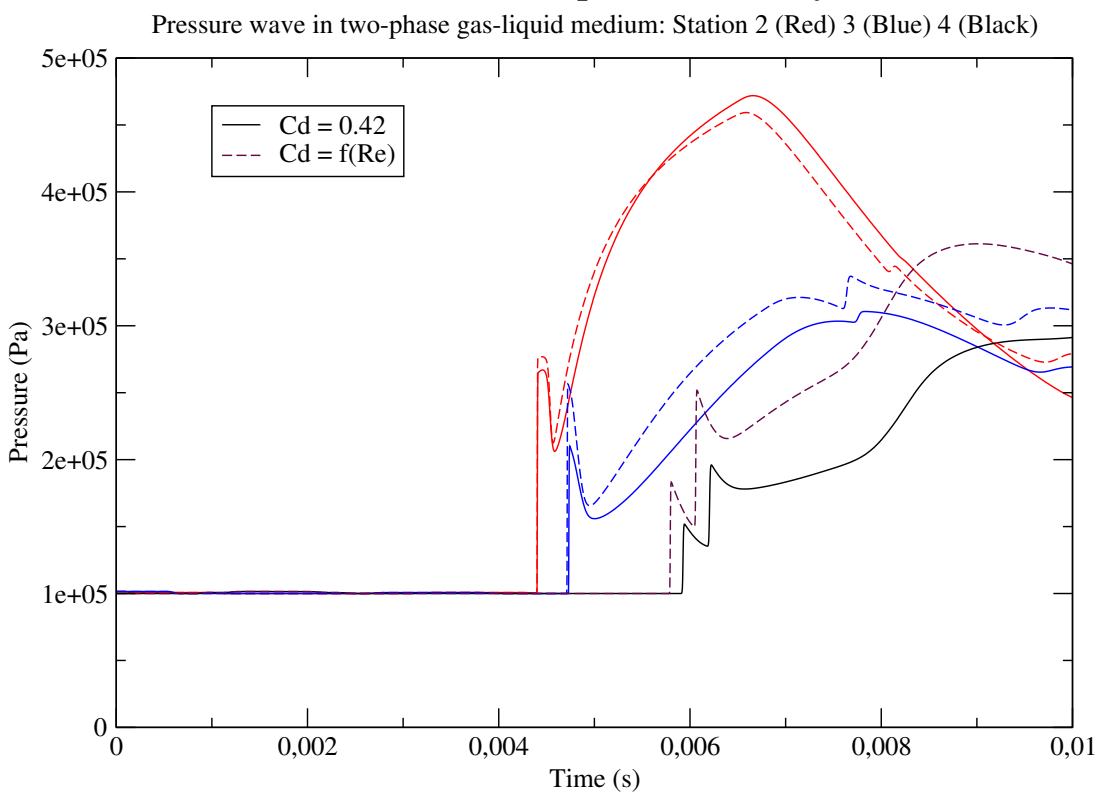

Fig. 16 Two-phase shock tube apparatus with liquid water droplets. Influence of the drag coefficient $C_{12}$ on the mean pressure profiles at stations $2-3-4$.

33. X. Rogue, G. Rodriguez, J.F. HaAs ANd R. SAUrel, Experimental and numerical investigation of the shock induced fluidization of a particles bed, Shock Waves, vol. 8, pp.29-46, 2014.

34. E. Romenski, A. A. Belozerov And I. M. Peshrov, Conservative formulation for compressible multiphase flows, http://arxiv.org/abs/1405.3456, pp. 1-21, 2014.

35. E. RUSANOv, Calculation of interaction of non steady shock waves with obstacles, Journal of Computational Mathematics and Physics, vol. 1, pp. 267-279, 1961.

36. K. SALEH, A relaxation scheme for a hyperbolic multiphase flow model. Part I: barotropic EOS, HAL preprint available on https: https://hal.archives-ouvertes.fr/hal-01737681v1, 2018.

37. W. YAO AND C. MOREL, Volumetric interfacial area prediction in upward bubbly two-phase flow, Int. J. of Heat and Mass Transfer, vol. 47, pp. 307-328, 2004. 